SFB 649 Discussion Paper 2014-046

\title{
Ex post information rents in sequential screening
}

\author{
Daniel Krähmer* \\ Roland Strausz**
}

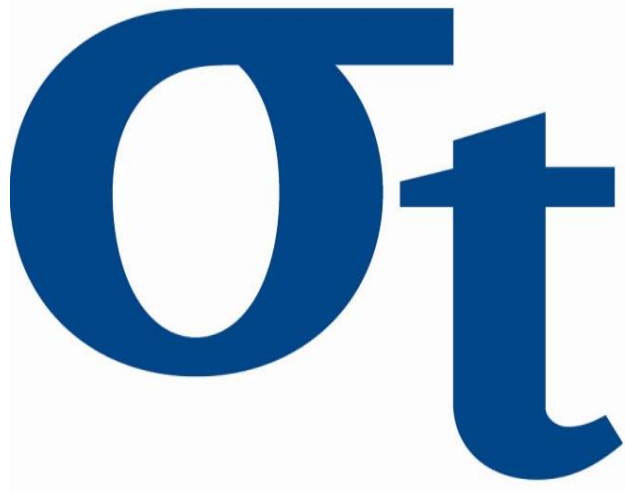

a

$\checkmark$

6

* Universität Bonn, Germany

** Humboldt-Universität zu Berlin, Germany

This research was supported by the Deutsche Forschungsgemeinschaft through the SFB 649 "Economic Risk".

http://sfb649.wiwi.hu-berlin.de ISSN 1860-5664 


\title{
Ex post information rents in sequential screening
}

\author{
Daniel Krähmer*and Roland Strausz ${ }^{\dagger}$
}

March 13, 2014

\begin{abstract}
We study ex post information rents in sequential screening models where the agent receives private ex ante and ex post information. The principal has to pay ex post information rents for preventing the agent to coordinate lies about his ex ante and ex post information. When the agent's ex ante information is discrete, these rents are positive, whereas they are zero in continuous models. Consequently, full disclosure of ex post information is generally suboptimal. Optimal disclosure rules trade off the benefits from adapting the allocation to better information against the effect that more information aggravates truth-telling.
\end{abstract}

Keywords: information rents, sequential screening, information disclosure JEL codes: D82, H57

${ }^{*}$ University Bonn, Department of Economics, Institute for Microeconomics, Adenauer Allee 24-42, D-53113 Bonn (Germany), kraehmer@hcm.uni-bonn.de.

${ }^{\dagger}$ Humboldt-Universität zu Berlin, Institute for Economic Theory 1, Spandauer Str. 1, D-10178 Berlin (Germany), strauszr@wiwi.hu-berlin.de. We thank Peter Esö, Philipp Kircher, R. Vijay Krishna, Alessandro Pavan, Balazs Szentes, and seminar audiences at Bocconi, Bonn, CESIfo Munich, Florence, Yale. We gratefully acknowledge financial support by the DFG (German Science Foundation) under SFB/TR-15 and SFB649. 


\section{Introduction}

Economic theory recognizes that private information is a crucial determinant of economic inefficiencies. In adverse selection models where a principal faces a privately informed agent at the contracting stage, such as in monopolistic price discrimination (e.g, Baron and Myerson, 1982) or public procurement (e.g. Laffont and Tirole, 1986), the agent's private information forces the seller to concede information rents to the agent. As a result, a trade-off between rent extraction and efficiency emerges which leads to economic inefficiencies.

In contrast, Harris and Raviv (1978) show that, when the agent's private information arrives only ex post, after the agent's decision to participate in the relationship, this trade-off between rent extraction and efficiency does not emerge: the principal can extract the full surplus from the relation without leaving rents to the agent. ${ }^{1}$ Esö and Szentes (2007a, b) extend this result to a framework where the agent receives both ex ante and ex post private information. In particular, they decompose the agent's final information into a piece that arrives ex ante and a piece that arrives ex post and is independent of the ex ante information. This formulation identifies the agent's ex post information with the additional information, also referred to as the "shock", that he receives beyond the information he already has ex ante. In this setting, Esö and Szentes (2007a, b) show that at the optimal mechanism, the agent's private knowledge of the shock does not add to the information rents that he receives already from his ex ante information. ${ }^{2}$ These results suggest the general insight that only ex ante private information is a source of information rents, whereas ex post private information is not.

The purpose of this paper is to qualify this insight and to offer a more comprehensive perspective of the role of private ex post private information in dynamic adverse selection problems. Our main contribution is to show that the insight of Harris and Raviv (1978) that the principal can extract all rents from ex post private information extends only to models in which the agent's ex ante private information is smooth and continuous such as Esö and Szentes (2007a,b). In contrast, the principal has to concede a strictly positive information rent for extracting the agent's ex post information when the agent's ex ante private information

\footnotetext{
${ }^{1}$ This holds in a framework with quasi-linear preferences where the agent is effectively risk neutral.

${ }^{2}$ This is shown in a setting with two periods and one allocation decision for a single agent by Esö and Szentes (2007a) and for the case with many agents by Esö and Szentes (2007b). Esö and Szentes (2013) generalizes the result to a setting with allocation decisions in multiple periods and moral hazard.
} 
is discrete. In order to demonstrate this result, we provide a formal decomposition of the agent's total information rents into a part that accrues from his ex ante information and a part that accrues from the privacy of the shock. Using this decomposition, we show that, in general, the seller has to concede to the agent additional rents for eliciting the shock, because the agent may benefit from coordinating lies about his ex ante information with lies about the realization of the shock. We show, however, that when the distance between ex ante types diminishes, the agent's potential benefits from coordinating his lies tend to zero at a faster speed than the distance between ex ante types. As a result, marginal ex post information rents vanish in the limit of a smooth continuous model. ${ }^{3}$

We further show that, as an implication of our result, full disclosure of ex post information is no longer generally optimal when the agent's ex ante private information is discrete, whereas, as shown by Esö and Szentes (2007a, b), full disclosure is optimal in continuous models. We identify two conflicting effects of disclosure. More disclosure is beneficial to the principal because it allows him to choose among a larger set of allocations, as allocations can indirectly depend on the agent's message about the disclosed information. The negative effect of more disclosure is however that, as already noted in Myerson (1991, p.297), "revealing more information to players makes it harder to prevent them from finding ways to gain by lying". The seller's optimal disclosure rule trades off these two effects.

This paper contributes to the literature on dynamic mechanism design by focusing on the role of dynamic private information. ${ }^{4}$ We built on Esö and Szentes' (2007a, b) approach to study the relevance of ex post information in models with both discrete and continuous ex ante information. Next to demonstrating the relevance of ex post information in discrete models, we provide an alternative proof for their result that ex post information is irrelevant in continuous models.

Two recent papers are closely related to our partial disclosure result. First, also Li and Shi (2013) present a partial disclosure result for the standard sequential screening model. The

\footnotetext{
${ }^{3}$ That information rents are zero in smooth continuous models is a consequence of the dynamic revenue equivalence property established in Pavan et al. (2013). When ex ante information is discrete, revenue equivalence fails, opening the door for the possibility that the agent gains from a lie about the shock after an initial lie.

${ }^{4}$ E.g., Baron and Besanko (1984), Courty and Li (2000), Battaglini (2005), Esö and Szentes (2007a, b), Hoffmann and Inderst (2011), Krähmer and Strausz (2011), Nocke et al. (2011), Pavan et al. (2013), Boleslavsky and Said (2013), Deb and Said (2013).
} 
key conceptual difference between our and their result is that Li and Shi (2013) consider more general disclosure rules. In their framework the seller can disclose any information about the final payoff type, which is a combination of both the buyer's initial private information and the shock. In contrast, we allow partial disclosure only of the shock. The results of Esö and Szentes (2007a) imply that with continuous type distributions, partial disclosure of the shock is never strictly optimal. Hence, whereas we show that this is no longer true with discrete types, Li and Shi (2013) show that partial disclosure may be strictly optimal if it concerns the final payoff type rather than the shock.

Second, Bergemann and Wambach (2013) provide an additional reason why partial disclosure might be optimal. They show for the setting of Esö and Szentes (2007a) that by only partially disclosing the additional information, i.e., the shock, the seller can relax participation constraints without impairing revenue. Hence, if the buyer must not make losses, conditional on the additional information disclosed, partial disclosure of the shock is optimal. In contrast, in our setting partial disclosure may be optimal even though the buyer can make losses ex post, after additional information has been disclosed.

The rest of the paper is organized as follows. We introduce the formal model in the next section. In Section 3, we set up the seller's problem for the case where the agent's ex post information is private and public and discuss how these two problems relate to the question of ex post information rents. In Section 4, we study the case of two ex ante types and demonstrate that positive ex post information rents are necessary to prevent coordinated lying. In Section 5, we study ex post information rents for the general case, distinguishing between smooth continuous models, where the ex post information rent vanishes, and discrete models, where it does not. In Section 6 we explain with an explicit example that the seller is strictly better off by disclosing ex post information only partially rather than fully. Section 7 concludes. All proofs are relegated to an appendix.

\section{The Model}

There is a seller (she) and a buyer (he). The seller's costs to produce the good are commonly known and normalized to zero. The buyer's valuation is $v$ and takes values in $[\underline{v}, \bar{v}]$. The terms of trade are the probability with which the good is sold, $x \in[0,1]$, and a payment $t \in \mathbb{R}$ from the buyer to the seller. Parties are risk-neutral and have quasi-linear utility functions. 
That is, under the terms of trade $x$ and $t$, the seller receives utility $t$, and the buyer receives utility $v x-t$. The seller's objective is to design a selling mechanism that maximizes her expected revenue where a selling mechanism specifies the terms of trade, possibly contingent on communication between the parties.

When the seller offers a mechanism to the buyer, no party knows the true valuation, $v$, but the buyer is better informed about the distribution from which $v$ is drawn than the seller. Formally, it is common knowledge that $v$ is distributed according to the distribution function $F(\cdot \mid \theta)$, where $\theta$ is drawn from the support $\Theta \subseteq \mathbb{R}$ with distribution function $P$. We follow the literature and assume "non-shifting support", that is, the support of $F(\cdot \mid \theta)$ is the interval $[\underline{v}, \bar{v}]$ for all $\theta \in \Theta$. The distributions $\{F(v \mid \theta) \mid \theta \in \Theta\}$ are ranked according to first order stochastic dominance, that is, $\theta>\hat{\theta}$ implies $F(v \mid \theta)<F(v \mid \hat{\theta})$ for all $v \in(\underline{v}, \bar{v})$. We further assume that the density $f(v \mid \theta)=\partial F(v \mid \theta) / \partial v$ exists for each $\theta \in \Theta$ and is bounded away from zero, i.e., there exists some $\varepsilon>0$ such that $f(v \mid \theta)>\varepsilon$ for all $v \in[\underline{v}, \bar{v}]$ and $\theta \in \Theta$. Hence, $F(v \mid \theta)$ is strictly increasing in $v$ and decreasing in $\theta$.

Before the seller offers the buyer a mechanism, the buyer privately observes the signal $\theta$ so that we interpret $\theta$ as the buyer's ex ante private information. If the buyer accepts the mechanism, then, before production takes place, $v$ is drawn according to $F(\cdot \mid \theta)$ and the buyer observes this realization privately. Because we are interested in the information rent the buyer can secure from observing additional private information ex post, we need a proper definition of the buyer's ex post private information.

It is important to note that while the buyer observes his valuation $v$ ex post, $v$ contains more than the additional information the buyer receives ex post. Intuitively, the buyer's additional information is the "difference" between the information contained in his valuation and his ex ante information. To capture this formally, we follow the idea of Esö and Szentes (2007a, b) and write the buyer's valuation as a compound of ex ante information and additional, orthogonal ex post information. Define the random variable $\sigma=F(v \mid \theta)$. Then $\sigma$ is uniformly distributed on the unit interval and is stochastically independent of $\theta .{ }^{5}$ The buyer's valuation can be backed out as a function of $\theta$ and $\sigma$ by

$$
v=v(\theta, \sigma) \equiv F^{-1}(\sigma \mid \theta)
$$

where $F^{-1}$ is the inverse of $F$ with respect to $v$ and exists because $F(v \mid \theta)$ is strictly increasing

\footnotetext{
${ }^{5}$ Note that for all $\theta$, it holds: $\operatorname{Prob}(\sigma<\bar{\sigma})=F\left(F^{-1}(\bar{\sigma} \mid \theta) \mid \theta\right)=\bar{\sigma}$.
} 
in $v$. Hence, instead of assuming that $\theta$ and $v$ is observed, we can equivalently assume that $\theta$ and $\sigma$ is observed. Because $\sigma$ is independent of $\theta$, the formulation in terms of $\theta$ and $\sigma$ allows us to interpret $\sigma$ as the agent's (additional) ex post private information. In line with standard terminology, we also refer to $\sigma$ as the shock.

Thus we consider the following timing:

1. The buyer privately observes $\theta$.

2. The seller offers a mechanism.

3. The buyer accepts or rejects.

If he rejects, both parties receive their outside option normalized to zero.

4. If the buyer accepts, the buyer privately observes $\sigma$.

5. The terms of trade are implemented according to the mechanism.

We now introduce a distinction which will be key for our analysis:

Definition 1 (a) We say that the model is continuous if $\Theta$ is an interval $[\underline{\theta}, \bar{\theta}]$ and for all $\theta \in(\underline{\theta}, \bar{\theta})$ :

$$
\frac{\partial F(v \mid \theta)}{\partial \theta} \text { exists and is bounded. }
$$

(b) We say that the model is discrete if $\Theta$ is a discrete set $\{\underline{\theta}, \ldots, \theta, \ldots, \bar{\theta}\} .{ }^{6}$

For the case that the model is continuous, our setting is identical to the setup in Esö and Szentes $(2007 \mathrm{a}, \mathrm{b})^{7}$ who show that the privacy of the ex post information $\sigma$ is irrelevant in the sense that the seller can fully extract the value of the buyer's ex post information. The main point of our paper is to qualify this result for the case that the model is discrete, and to identify the source of the buyer's ex post information rent. To make this point, we follow Esö and Szentes (2007a, b) and compare the optimal selling mechanism when $\sigma$ is observed privately by the buyer to the optimal mechanism in the benchmark case when $\sigma$ is publicly observable.

\footnotetext{
${ }^{6}$ Alternatively, we could equivalently define a discrete model as a model in which $\Theta$ is an interval and $F(v \mid \theta)$ is piece-wise constant in $\theta$. Clearly, then $F(v \mid \theta)$ would not be differentiable for some $\theta \in \Theta$ and the model would not be continuous in our sense. We believe that our formulation is more in line with standard practice in mechanism design.

${ }^{7}$ Esö and Szentes (2007b) consider the auction case when the seller faces multiple potential buyers. Allowing for multiple buyers would not change our results.
} 
In what follows, it will often be more convenient to work with the function $v(\theta, \sigma)$ rather than with $F(v \mid \theta)$. Our assumptions on $F(v \mid \theta)$ translate directly into the following properties of $v(\theta, \sigma)$.

Lemma 1 (a) For all $\theta>\hat{\theta}$ :

$$
v(\theta, \sigma)>v(\hat{\theta}, \sigma) \quad \forall \sigma \in(0,1) .
$$

(b) For all $\theta, \hat{\theta} \in \Theta$ and $\sigma \in[0,1]$, there exists a unique $\sigma^{*} \in[0,1]$ such that

$$
v\left(\hat{\theta}, \sigma^{*}\right)=v(\theta, \sigma) .
$$

(c) For all $\theta \in \Theta, \sigma \in(0,1)$ :

$$
\frac{\partial v(\theta, \sigma)}{\partial \sigma} \text { exists, is strictly positive, and bounded. }
$$

(d) If the model is continuous, then for all $\theta \in \Theta, \sigma \in(0,1)$ :

$$
\frac{\partial v(\theta, \sigma)}{\partial \theta} \text { exists and is bounded. }
$$

\section{The seller's problem}

In describing the seller's problem, we begin with the case that the shock $\sigma$ is the buyer's private information, and then describe the case when the shock is publicly observable.

\subsection{Private shock}

When both ex ante information and the shock are the buyer's private information, we can apply the revelation principle for dynamic games (Myerson, 1986) which states that the optimal mechanism is in the class of direct and incentive compatible mechanisms. A direct and incentive compatible mechanism specifies the terms of trade as functions of reports by the buyer about his ex ante information and the shock and, moreover, induces the buyer, on the equilibrium path, to report his information truthfully.

Formally, a direct mechanism $(x, t)$ is a combination of an allocation rule $x$ and a transfer schedule $t$ with

$$
x: \Theta \times[0,1] \rightarrow[0,1], \quad t: \Theta \times[0,1] \rightarrow \mathbb{R}
$$


that requires the buyer to report a message $\hat{\theta} \in \Theta$ at the outset (before having observed $\sigma$ ) and to subsequently (after having observed $\sigma$ ) report a message $\hat{\sigma} \in[0,1]$. If a buyer who has observed $\theta$ and $\sigma$ reports $\hat{\theta}$ and $\hat{\sigma}$, his utility from the mechanism is

$$
u(\hat{\theta}, \hat{\sigma} ; \theta, \sigma)=v(\theta, \sigma) x(\hat{\theta}, \hat{\sigma})-t(\hat{\theta}, \hat{\sigma}) .
$$

With slight abuse of notation, we define the buyer's utility from truth-telling as $u(\theta, \sigma)=$ $u(\theta, \sigma ; \theta, \sigma)$.

We say the mechanism $(x, t)$ is ex post incentive compatible if it induces the buyer to report the shock truthfully after having truthfully reported his ex ante information:

$$
u(\theta, \sigma) \geq u(\theta, \hat{\sigma} ; \theta, \sigma) \quad \forall \theta, \sigma, \hat{\sigma} .
$$

Note that the revelation principle requires truth-telling in the second stage only after truthtelling in the first stage (see Myerson, 1986). Hence, it might (in fact, it will) be optimal for the buyer, after he lied about $\theta$ to lie also about $\sigma$. Thus, the buyer's expected utility from reporting $\hat{\theta}$ at the initial stage when he has observed $\theta$ is

$$
U(\hat{\theta} ; \theta)=\int_{0}^{1} \max _{\hat{\sigma}} u(\hat{\theta}, \hat{\sigma} ; \theta, \sigma) d \sigma .
$$

The "max" operator under the integral accounts for the fact that the mechanism may induce the buyer to lie about $\sigma$ after lying about $\theta$. Again abusing notation, we define the buyer's utility from truth-telling at the initial stage as $U(\theta)=U(\theta ; \theta)$.

We say that the mechanism is ex ante incentive compatible if it induces the buyer to report his ex ante information truthfully:

$$
U(\theta) \geq U(\hat{\theta} ; \theta) \quad \forall \theta, \hat{\theta} .
$$

A mechanism $(x, t)$ is incentive compatible with private shock if it is ex ante and ex post incentive compatible.

We also assume that the seller wants to ensure the buyer's participation in the mechanism, and therefore the mechanism needs to give the buyer his outside option of zero. A mechanism is individually rational with private shock if:

$$
U(\theta) \geq 0 \quad \forall \theta
$$


A mechanism is feasible with private shock if it is incentive compatible and individually rational with private shock. We say that the allocation rule $x$ is implementable with private shock if there are transfers $t$ so that $(x, t)$ is feasible with private shock. We define by ${ }^{8}$

$$
W(x)=\max _{t} \int_{S} \int_{0}^{1} t(\theta, \sigma) d \sigma d P(\theta) \quad \text { s.t. (9), (11), (12) }
$$

the principal's maximal expected revenue from an implementable allocation rule $x$.

The seller's problem with private shock, referred to as $\mathscr{P}$, is to choose a feasible mechanism that maximizes her expected revenue:

$$
\mathscr{P}: \quad \quad \quad \max _{x, t} \int_{S} \int_{0}^{1} t(\theta, \sigma) d \sigma d P(\theta) \text { s.t. (9), (11), (12). }
$$

We refer to a solution to $\mathscr{P}$ as $\left(x^{*}, t^{*}\right)$ and denote the seller's value from problem $\mathscr{P}$ by $W^{*}$.

\subsection{Publicly observable shock}

When the shock is publicly observable, the only private information of the buyer is his initial information $\theta$. The revelation principle then implies that the optimal selling mechanism is a direct and incentive compatible mechanism $(\tilde{x}, \tilde{t})$ which requires the buyer to report a message $\hat{\theta} \in \Theta$ about his ex ante information only, and which induces the buyer to report truthfully. In addition to the buyer's report, the mechanism can condition directly on the shock, as it is contractible by assumption.

Formally, if the buyer has observed $\theta$ and reports $\hat{\theta}$ at the outset, his expected utility from the mechanism $(\tilde{x}, \tilde{t})$ is

$$
\tilde{U}(\hat{\theta} ; \theta)=\int_{0}^{1}\{v(\theta, \sigma) \tilde{x}(\hat{\theta}, \sigma)-\tilde{t}(\hat{\theta}, \sigma)\} d \sigma .
$$

Note that, whatever the buyer's report $\hat{\theta}$, the terms of trade are enforced according to the true value of $\sigma$, as now $\sigma$ does not need to be elicited from the buyer any more. We define, again with slight abuse of notation, $\tilde{U}(\theta)=\tilde{U}(\theta ; \theta)$.

We say that a mechanism $(\tilde{x}, \tilde{t})$ is incentive compatible with public shock if it induces the buyer to report his initial information truthfully:

$$
\tilde{U}(\theta) \geq \tilde{U}(\hat{\theta} ; \theta) \quad \forall \theta, \hat{\theta} .
$$

\footnotetext{
${ }^{8}$ If $\Theta$ is discrete, the integral is to be understood as an integral over the discrete measure $P$ and is thus a sum.
} 
As before, the seller wants to ensure the buyer's participation in the mechanism, and therefore the mechanism needs to give the buyer his outside option of zero. We say a mechanism is individually rational with public shock if:

$$
\tilde{U}(\theta) \geq 0 \quad \forall \theta .
$$

A mechanism is feasible with public shock if it is incentive compatible and individually rational with public shock. We say that the allocation rule $\tilde{x}$ is implementable with public shock if there are transfers $\tilde{t}$ so that $(\tilde{x}, \tilde{t})$ is feasible with public shock. We define by

$$
\tilde{W}(\tilde{x})=\max _{\tilde{t}} \int_{\Theta} \int_{0}^{1} \tilde{t}(\theta, \sigma) d \sigma d P(\theta) \quad \text { s.t. } \quad(16),(17)
$$

the principal's maximal expected revenue from an implementable allocation rule $\tilde{x}$.

The seller's problem with public shock, referred to as $\tilde{\mathscr{P}}$, is to choose a feasible mechanism that maximizes her expected revenue:

$$
\tilde{\mathscr{P}}: \quad \quad \quad \max _{\tilde{x}, t} \int_{\Theta} \int_{0}^{1} \tilde{t}(\theta, \sigma) d \sigma d P(\theta) \text { s.t. (16), (17). }
$$

We refer to a solution to $\tilde{\mathscr{P}}$ as $\left(\tilde{x}^{*}, \tilde{t}^{*}\right)$ and denote the seller's value from problem $\tilde{\mathscr{P}}$ by $\tilde{W}^{*}$.

\subsection{Ex post information rents and revenue-irrelevance}

A direct comparison of the cases with private and public shock allows us to study the role of the privacy of ex post information in the design of the optimal mechanism. Clearly, a given allocation rule $x$ that is implementable with private shock is implementable with public shock, and, moreover, the seller obtains a (weakly) higher revenue from implementing $x$ when the shock is public. If the seller can attain the same revenue when the shock is private, then the seller does not need to leave any rent for eliciting the buyer's ex post information when she wants to implement $x$.

Moreover, comparing the values $W^{*}$ and $\tilde{W}^{*}$ reveals how the privacy of the shock bears on the seller's ability to extract surplus. If the seller attains the same revenue when $\sigma$ is private as when $\sigma$ is public information, then private ex post information is irrelevant in the sense that it does not affect inefficiencies. These considerations motivate the following definitions. 
Definition 2 (a) Let $x$ be implementable with private shock. We say that $x$ is implementable without ex post information rents if $W(x)=\tilde{W}(x)$.

(b) Private ex post information is said to be revenue-irrelevant if $W^{*}=\tilde{W}^{*}$.

Private ex post information may fail to be revenue-irrelevant for two reasons. First, the allocation rule $\tilde{x}^{*}$ that is optimal with public shock may simply not be implementable with private shock. Second, even if $\tilde{x}^{*}$ is implementable, it may not be implementable without ex post information rents.

Esö and Szentes (2007a, b) have shown that if the model is continuous and $\tilde{x}^{*}$ is implementable with private shock, then private ex post information is revenue-irrelevant. As a consequence, $\tilde{x}^{*}$ is implementable without ex post information rents. In what follows, we generalize the result of Esö and Szentes (2007a, b) and show that in continuous models, any allocation rule that is implementable with private shock is implementable without ex post information rents. More interestingly, we also show that this result is not true for discrete models. Indeed, in discrete models, an allocation rule that is implementable with private shock is implementable without ex post information rents only in degenerate cases. This directly implies that in discrete models, ex post information is revenue-irrelevant only in degenerate cases. In particular, even if the allocation rule $\tilde{x}^{*}$ is implementable with private shock, ex post information is typically not revenue-irrelevant.

To illustrate the basic features of our general analysis, we now analyze a discrete model with two types and show that private ex post information is not revenue-irrelevant.

\section{Two ex ante types}

Let $\Theta=\left\{\theta_{L}, \theta_{H}\right\}$ be the set of the buyer's ex ante types, occurring with $P\left(\theta_{L}\right)=1-p \in$ $(0,1)$ and $P\left(\theta_{H}\right)=p$. To simplify notation, we replace the function arguments $\theta_{L}$ and $\theta_{H}$ by appending function symbols with the subindices $L$ and $H$. We assume that type $\theta_{L}$ corresponds to the low valuation type and type $\theta_{H}$ to the high valuation type:

$$
v_{L}(\sigma)<v_{H}(\sigma) \quad \forall \sigma \in(0,1) .
$$




\subsection{Public shock}

We begin by deriving the optimal selling mechanism with public shock. With two ex ante types, a selling mechanism is a quadruple $(\tilde{x}, \tilde{t})=\left(\tilde{x}_{L}(\sigma), \tilde{t}_{L}(\sigma), \tilde{x}_{H}(\sigma), \tilde{t}_{H}(\sigma)\right)$, and the incentive compatibility and participation constraints (16) and (17) write

$$
\begin{aligned}
& I C_{H}: \quad \int_{0}^{1}\left\{v_{H}(\sigma) \tilde{x}_{H}(\sigma)-\tilde{t}_{H}(\sigma)\right\} d \sigma \geq \int_{0}^{1}\left\{v_{H}(\sigma) \tilde{x}_{L}(\sigma)-\tilde{t}_{L}(\sigma)\right\} d \sigma ; \\
& I C_{L}: \quad \int_{0}^{1}\left\{v_{L}(\sigma) \tilde{x}_{L}(\sigma)-\tilde{t}_{L}(\sigma)\right\} d \sigma \geq \int_{0}^{1}\left\{v_{L}(\sigma) \tilde{x}_{H}(\sigma)-\tilde{t}_{H}(\sigma)\right\} d \sigma ; \\
& I R_{H}: \quad \int_{0}^{1}\left\{v_{H}(\sigma) \tilde{x}_{H}(\sigma)-\tilde{t}_{H}(\sigma)\right\} d \sigma \geq 0 \\
& I R_{L}: \quad \int_{0}^{1}\left\{v_{L}(\sigma) \tilde{x}_{L}(\sigma)-\tilde{t}_{L}(\sigma)\right\} d \sigma \geq 0 .
\end{aligned}
$$

Standard arguments can be employed to show that at the optimal selling mechanism the incentive constraint for the high valuation type and the participation constraint for the low valuation type are binding and that the allocation rule has to satisfy a monotonicity property with respect to the ex ante information. ${ }^{9}$

Lemma 2 With two ex ante types, the mechanism $(\tilde{x}, \tilde{t})$ which is optimal with public shock maximizes the seller's objective subject to $\left(I C_{H}\right)$ and $\left(\operatorname{IR}_{L}\right)$ being binding, and $\int_{0}^{1}\left[v_{H}(\sigma)-v_{L}(\sigma)\right]\left[\tilde{x}_{H}(\sigma)-\right.$ $\left.\tilde{x}_{L}(\sigma)\right] d \sigma \geq 0$.

The binding constraints pin down the (expected) transfers as a function of the allocation rule according to

$$
\begin{aligned}
& \int_{0}^{1} \tilde{t}_{L}(\sigma) d \sigma=\int_{0}^{1} v_{L}(\sigma) \tilde{x}_{L}(\sigma) d \sigma \\
& \int_{0}^{1} \tilde{t}_{H}(\sigma) d \sigma=\int_{0}^{1} v_{H}(\sigma) \tilde{x}_{H}(\sigma) d \sigma+\int_{0}^{1}\left[v_{L}(\sigma)-v_{H}(\sigma)\right] \tilde{x}_{L}(\sigma) d \sigma .
\end{aligned}
$$

Substituting out the transfer schedules in the seller's objective, the seller's problem simplifies to

$$
\begin{aligned}
\max _{\tilde{x}, \tilde{t}} & \int_{0}^{1}\left\{p v_{H}(\sigma) \tilde{x}_{H}(\sigma)+\left[v_{L}(\sigma)-p v_{H}(\sigma)\right] \tilde{x}_{L}(\sigma)\right\} d \sigma \\
& \text { s.t. } \quad \int_{0}^{1}\left[v_{H}(\sigma)-v_{L}(\sigma)\right]\left[\tilde{x}_{H}(\sigma)-\tilde{x}_{L}(\sigma)\right] d \sigma \geq 0 .
\end{aligned}
$$

${ }^{9}$ The condition $\int_{0}^{1}\left[v_{H}(\sigma)-v_{L}(\sigma)\right]\left[\tilde{x}_{H}(\sigma)-\tilde{x}_{L}(\sigma)\right] d \sigma \geq 0$ is a monotonicity condition because, as $v_{H}(\sigma)-$ $v_{L}(\sigma)>0$ for all $\sigma \in(0,1)$, the condition is satisfied if $\tilde{x}_{H}(\sigma) \geq \tilde{x}_{L}(\sigma)$ "on average". 
To solve this problem, we can adopt the standard approach and first ignore the monotonicity constraint (27). We then obtain the solution to this relaxed problem by point-wise maximization. It is then easy to verify that the solution to the relaxed problem always satisfies the monotonicity constraint so that it is also a solution to the original problem. We summarize this in the next lemma.

Lemma 3 With two types, the solution $\left(\tilde{x}^{*}, \tilde{t}^{*}\right)$ to problem $\tilde{\mathscr{P}}$ is given by

$$
\tilde{x}_{H}^{*}(\sigma)=1 \quad \forall \sigma \in[0,1], \quad \tilde{x}_{L}^{*}(\sigma)= \begin{cases}0 & \text { if } \quad v_{L}(\sigma)<p v_{H}(\sigma) \\ 1 & \text { else. }\end{cases}
$$

The optimal transfers $\tilde{t}_{H}^{*}$ and $\tilde{t}_{L}^{*}$ are only pinned down in expectation by (25) and (26).

Thus, under the optimal mechanism with public shock, the high valuation type $\theta_{H}$ always consumes the good ("no distortion at the top"), and the low valuation type $\theta_{L}$ consumes the good only if $v_{L}(\sigma) \geq p v_{H}(\sigma)$. The high valuation type $\theta_{H}$ obtains an information rent of

$$
\tilde{U}_{H}=\int_{0}^{1}\left[v_{H}(\sigma)-v_{L}(\sigma)\right] \tilde{x}_{L}^{*}(\sigma) d \sigma=\int_{\sigma: v_{L}(\sigma) \geq p v_{H}(\sigma)}\left\{v_{H}(\sigma)-v_{L}(\sigma)\right\} d \sigma .
$$

\subsection{Private shock}

We now show that when the shock is private, the seller is strictly worse off than when the shock is public. We derive this result by showing that, with private shock, the seller has to concede to the buyer strictly more information rents if she wants to implement the same allocation rule $\tilde{x}^{*}$ that is optimal with public shock. That is, $\tilde{x}^{*}$ is not implementable without ex post information rents.

To prove our claim, we can use the following result from the literature: a deterministic allocation rule ${ }^{10}$ is implementable with private shock if, and only if, two properties hold: first, for each ex ante type, the allocation rule is non-decreasing in $\sigma$; second, if the allocation rule prescribes consumption at a certain valuation for some ex ante type, then consumption is prescribed at all higher valuations for all larger ex ante types. ${ }^{11,12}$ We now apply this result to the allocation rule $\tilde{x}^{*}$ in (28). Note that the second requirement is trivially met since

\footnotetext{
${ }^{10}$ An allocation rule $x$ is deterministic if $x(\theta, \sigma) \in\{0,1\}$ for all $\theta$ and $\sigma$.

${ }^{11}$ Formally: for $\theta<\hat{\theta}$, if $x(\theta, \sigma)=1$, then $x(\hat{\theta}, \hat{\sigma})=1$ for all $\hat{\sigma}$ with $v(\hat{\theta}, \hat{\sigma}) \geq v(\theta, \sigma)$.

${ }^{12}$ The "if" part holds more generally also for non-deterministic allocation rules and was first proven in Courty and Li (2000). For a proof of the "only if" part, see Krähmer and Strausz (2011, Lemma 6).
} 
$\tilde{x}_{H}^{*}(\sigma)=1$ for all $\sigma$. The first requirement is met for type $\theta=\theta_{H}$ since $\tilde{x}_{H}^{*}(\sigma)$ is clearly non-decreasing in $\sigma$. Thus, all that is needed to implement $\tilde{x}^{*}$ is that $\tilde{x}_{L}^{*}$ is non-decreasing in $\sigma$ :

Lemma 4 The allocation rule $\tilde{x}^{*}$ in (28) is implementable with private shock if, and only if, $\tilde{x}_{L}^{*}(\sigma)$ is non-decreasing in $\sigma$.

Hence, if $\tilde{x}_{L}^{*}$ is not non-decreasing, private ex post information is revenue-relevant for the simple reason that the optimal allocation rule $\tilde{x}^{*}$ is not implementable with private shock. More interestingly, we now show that even if $\tilde{x}^{*}$ is implementable with private shock, private ex post information is not revenue-irrelevant. Therefore, we focus from now on on the case that $\tilde{x}_{L}^{*}$ is monotone in $\sigma$. A sufficient condition for this is that the ratio $v_{L}(\sigma) / v_{H}(\sigma)$ is strictly increasing because this guarantees a unique solution $\sigma_{L}$ to the equation $v_{L}\left(\sigma_{L}\right)=p v_{H}\left(\sigma_{L}\right)$, and (28) then prescribes the good to be sold to the low valuation buyer type whenever $\sigma \geq$ $\sigma_{L} \cdot{ }^{13}$ We now show that to implement $\tilde{x}^{*}$ with private shock, the seller has to concede a higher information rent to the buyer than in the case with public shock.

Indeed, ex post incentive compatibility requires that the buyer type $\theta_{L}$, after having reported his ex ante information truthfully, reports $\sigma$ truthfully in the second stage. This implies that the transfers $t_{L}$ are piece-wise constant, and that at the critical realization $\sigma_{L}$, the buyer type $\theta_{L}$ is indifferent between consuming and not consuming the good:

$$
t_{L}(\sigma)=\left\{\begin{array}{ll}
t_{L}^{0} & \text { if } \sigma<\sigma_{L} \\
t_{L}^{1} & \text { else }
\end{array}, \text { and }-t_{L}^{0}=v_{L}\left(\sigma_{L}\right)-t_{L}^{1} .\right.
$$

To see that ex post incentive compatibility implies these restrictions on the transfer schedule, note that if $t_{L}$ were not constant on $\left[0, \sigma_{L}\right)$, then since the allocation rule is constant on $\left[0, \sigma_{L}\right)$, there would be some realizations in $\left[0, \sigma_{L}\right)$ for which the buyer would benefit from reporting the value of $\sigma$ that maximizes $t_{L}$ on $\left[0, \sigma_{L}\right)$ instead of reporting his true value. For the same reason, $t_{L}$ needs to be constant on $\left[\sigma_{L}, 1\right]$. On a related note, if the buyer were not indifferent between consuming and not consuming the good at the value $\sigma_{L}$, but, say, strictly preferred consumption, then he would still strictly prefer consumption at a value $\sigma$ slightly smaller than $\sigma_{L}$ and would thus benefit from pretending to have observed $\sigma_{L}$ in this case.

Condition (30) pins down only the difference $t_{L}^{1}-t_{L}^{0}=v_{L}\left(\sigma_{L}\right)$, but not the absolute magnitude of the transfers. As is usual, we may interpret $r_{L} \equiv t_{L}^{1}-t_{L}^{0}$ as an exercise price at

\footnotetext{
${ }^{13}$ An example for which $v_{L}(\sigma) / v_{H}(\sigma)$ is strictly increasing, is: $v_{H}(\sigma)=\sigma$, and $v_{L}(\sigma)=\sigma^{2}$.
} 
which the buyer is allowed to buy the product after learning $\sigma$. Likewise, $t_{L}^{0}$ corresponds to an upfront payment by the buyer that gives him the option to make his consumption decision after he has fully learned his valuation. Note that by adjusting the upfront payment $t_{L}^{0}$, while keeping the exercise price fixed, the seller determines the buyer's utility without affecting the allocation. The expected utility of the buyer type $\theta_{L}$ if he reports his ex ante information truthfully is

$$
U_{L}=-t_{L}^{0}+\int_{\sigma_{L}}^{1}\left\{v_{L}(\sigma)-r_{L}\right\} d \sigma .
$$

Now observe that $\tilde{x}_{L}^{*}$ and the payments $t_{L}$ fully determine the high valuation buyer's expected utility $U_{L H}$ when he pretends to be type $\theta_{L}$. Since ex ante incentive compatibility requires that the high valuation buyer attains at least a rent of $U_{L H}$, we can now obtain a lower bound on the rent that the seller has to concede to the buyer. To compute $U_{L H}$, note that after having reported $\theta_{L}$, the buyer type $\theta_{H}$ exercises the option whenever

$$
v_{H}(\sigma)>r_{L} \Leftrightarrow \sigma>v_{H}^{-1}\left(r_{L}\right) .
$$

The key observation is that when the high valuation type $\theta_{H}$ reports $\theta_{L}$, he exercises the option more frequently than when the low valuation type $\theta_{L}$ reports $\theta_{L}$. This is simply so because for any realization $\sigma$ of the shock, he displays a higher valuation than the buyer type $\theta_{L}$. Hence, for any value of $\sigma$ so that $v_{H}(\sigma)>r_{L}>v_{L}(\sigma)$ the buyer type $\theta_{H}$ does, and the buyer type $\theta_{L}$ does not exercise the option. Therefore,

$$
\begin{aligned}
U_{L H} & =-t_{L}^{0}+\int_{v_{H}^{-1}\left(r_{L}\right)}^{1}\left\{v_{H}(\sigma)-r_{L}\right\} d \sigma \\
& =-t_{L}^{0}+\int_{v_{H}^{-1}\left(r_{L}\right)}^{\sigma_{L}}\left\{v_{H}(\sigma)-r_{L}\right\} d \sigma+\int_{\sigma_{L}}^{1}\left\{v_{H}(\sigma)-r_{L}\right\} d \sigma \\
& =U_{L}+\int_{v_{H}^{-1}\left(r_{L}\right)}^{\sigma_{L}}\left\{v_{H}(\sigma)-r_{L}\right\} d \sigma+\int_{\sigma_{L}}^{1}\left\{v_{H}(\sigma)-v_{L}(\sigma)\right\} d \sigma,
\end{aligned}
$$

where we have used (31) in the last line.

To obtain a lower bound on the rent the seller has to concede to the high valuation buyer, note that ex ante incentive compatibility requires $U_{H} \geq U_{L H}$ and individual rationality requires $U_{L} \geq 0$. As a result, by (33), the high valuation type $\theta_{H}$ obtains a rent of at least ${ }^{14}$

$$
U_{H} \geq \int_{v_{H}^{-1}\left(r_{L}\right)}^{\sigma_{L}}\left\{v_{H}(\sigma)-r_{L}\right\} d \sigma+\int_{\sigma_{L}}^{1}\left\{v_{H}(\sigma)-v_{L}(\sigma)\right\} d \sigma .
$$

\footnotetext{
${ }^{14}$ It may be shown that this lower bound is actually attainable.
} 
Now, reconsider the high valuation type's rent when the shock $\sigma$ is public. For the case that $\tilde{x}_{L}$ is monotone, we obtain from (29):

$$
\tilde{U}_{H}=\int_{\sigma_{L}}^{1}\left\{v_{H}(\sigma)-v_{L}(\sigma)\right\} d \sigma .
$$

Comparing expression (34) to expression (35) makes clear that the buyer obtains an additional information rent from the privacy of the shock of

$$
\Delta U_{H}=U_{H}-\tilde{U}_{H} \geq \int_{v_{H}^{-1}\left(r_{L}\right)}^{\sigma_{L}}\left\{v_{H}(\sigma)-r_{L}\right\} d \sigma .
$$

This expression also identifies the source of this additional rent. With public shock, the contract enforces consumption directly, and irrespective of his true valuation, the buyer consumes only for $\sigma \geq \sigma_{L}$. In contrast, with private shock, the buyer effectively makes the consumption decision himself. As a result of his higher valuation, the high valuation buyer consumes the good more frequently than the low valuation buyer, and this is an additional source of rents: relative to the low valuation buyer, the high valuation buyer obtains not only the rent $v_{H}(\sigma)-v_{L}(\sigma)$ whenever both buyer types decide to consume but he also gets the rent $v_{H}(\sigma)-r_{L}$ when the high valuation buyer does and the low valuation buyer does not decide to consume ex post.

More generally, the transfers $\tilde{t}^{*}$ that prevent the buyer from lying when the shock is public are not sufficient to prevent the buyer from lying when the shock is private. This is so because when the shock is private, a lie about his ex ante information is more valuable to the buyer since he can coordinate it with a lie about the shock. Put differently, while $\tilde{x}^{*}$ is implementable with private shock "on the path", it is not implementable with private shock "off the path" after a lie by the high valuation buyer. Even though a lie by the buyer is a zero probability event, the fact that the buyer can use his private ex post information to obtain a strictly higher utility off the path relative to when the shock is public implies that the seller has to pay a higher rent to prevent the buyer from lying than with public shock.

To see that the strictly higher rents imply that private ex post information is not revenueirrelevant, observe that $\tilde{x}^{*}$ is the unique optimal allocation rule when the shock is public. Therefore, implementing any different allocation rule $x^{*} \neq \tilde{x}^{*}$ yields a smaller payoff than $\tilde{W}^{*}$ already with public shock. Because, as shown, $\tilde{x}^{*}$ requires the seller to pay larger rents to the buyer, the seller's revenue with private shock is smaller than $\tilde{W}^{*}$ so that private ex post information is revenue-relevant. 


\section{The general case}

In this section, we generalize the findings for the two types case. We first provide a decomposition of the buyer's rent into ex ante and ex post information rent. This is the crucial step to study the role of ex post information.

\subsection{Ex ante and ex post information rents}

We begin with two auxiliary lemmas. The first lemma states the well-known characterization of ex post incentive compatibility (we omit the proof).

Lemma 5 The mechanism $(x, t)$ is ex post incentive compatible if, and only if, $u(\theta, \sigma)$ is absolutely continuous in $\sigma$, and it holds for all $\theta \in \Theta$ :

$$
\begin{aligned}
& \frac{\partial u(\theta, \sigma)}{\partial \sigma}=\frac{\partial v(\theta, \sigma)}{\partial \sigma} x(\theta, \sigma) \text { for almost all } \sigma, \\
& x(\theta, \sigma) \text { is non-decreasing in } \sigma .
\end{aligned}
$$

We next characterize the buyer's ex post reporting strategy. We define by

$$
\Gamma^{*}(\theta, \hat{\theta}, \sigma)=\arg \max _{\hat{\sigma}} u(\hat{\theta}, \hat{\sigma} ; \theta, \sigma)
$$

all optimal second stage reports by a buyer who has observed $\theta$ and $\sigma$ and who has reported $\hat{\theta}$ at the outset. The next lemma describes how the buyer optimally coordinates a lie about his ex ante information with a lie about the shock. It says that a buyer type who has observed $\theta$ and $\sigma$ and who has reported $\hat{\theta}$ submits a "corrected" report $\sigma^{*}$ so that he has the same valuation and ends up with the same utility as a buyer type who has observed $\hat{\theta}$ and $\sigma^{*}$ and who has reported truthfully.

Lemma 6 If a mechanism is ex post incentive compatible, then there is a $\sigma^{*}=\sigma^{*}(\theta, \hat{\theta}, \sigma) \in$ $\Gamma^{*}(\theta, \hat{\theta}, \sigma)$ so that:

(a) $v(\theta, \sigma)=v\left(\hat{\theta}, \sigma^{*}\right)$;

(b) $u\left(\hat{\theta}, \sigma^{*} ; \theta, \sigma\right)=u\left(\hat{\theta}, \sigma^{*}\right)=\max _{\hat{\sigma}} u(\hat{\theta}, \hat{\sigma} ; \theta, \sigma)$.

In light of the lemma, we can, without loss of generality, assume that a buyer who has observed $\theta$ and $\sigma$ and who has reported $\hat{\theta}$ at the outset, reports $\sigma^{*}=\sigma^{*}(\theta, \hat{\theta}, \sigma)$ in the second stage. 
We can now provide a decomposition of the buyer's information rent in ex ante and ex post rent. For an arbitrary allocation rule $x$, let

$$
\begin{aligned}
& R^{x a}(\theta, \hat{\theta}, x) \equiv \int_{0}^{1}[v(\theta, \sigma)-v(\hat{\theta}, \sigma)] x(\hat{\theta}, \sigma) d \sigma \\
& R^{x p}(\theta, \hat{\theta}, x) \equiv \int_{0}^{1} \int_{\sigma}^{\sigma^{*}(\theta, \hat{\theta}, \sigma)} \frac{\partial v(\hat{\theta}, c)}{\partial \sigma}[x(\hat{\theta}, c)-x(\hat{\theta}, \sigma)] d c d \sigma .
\end{aligned}
$$

Lemma 7 (a) A mechanism $(\tilde{x}, \tilde{t})$ is incentive compatible with public shock if, and only if, for all $\theta, \hat{\theta}$ :

$$
\tilde{U}(\theta)-\tilde{U}(\hat{\theta}) \geq R^{x a}(\theta, \hat{\theta}, \tilde{x})
$$

(b) A mechanism $(x, t)$ is incentive compatible with private shock if, and only if, (37) and (38) are satisfied and for all $\theta, \hat{\theta}$, it holds:

$$
U(\theta)-U(\hat{\theta}) \geq R^{x a}(\theta, \hat{\theta}, x)+R^{x p}(\theta, \hat{\theta}, x) .
$$

Moreover, $R^{x p}(\theta, \hat{\theta}, x) \geq 0$, where the inequality is strict if $x(\hat{\theta}, \sigma)$ is not constant in $\sigma$ on $(0,1)$.

Lemma 7 provides, for both the case with private and public shock, lower bounds for the information rents a buyer of type $\theta$ has to obtain so as not to pretend to be of type $\hat{\theta}$. When the shock is private, the lower bound can be decomposed into a part $R^{x a}$ and a part $R^{x p}$. The part $R^{x a}$ is the same as the lower bound when ex post information is public and, therefore, accrues from the buyer's ex ante private information. Consequently, the part $R^{x p}$ is the buyer's rent that accrues from observing the shock privately.

Figure 1 illustrates the decomposition graphically for the two types case and the allocation rule $\tilde{x}^{*}$ given by (28) when $\tilde{x}_{L}^{*}$ is monotone. When the shock is public, the high valuation buyer type $\theta_{H}$ can, by announcing type $\hat{\theta}=\theta_{L}$, secure himself the same utility as the low valuation buyer plus the utility increment $v_{H}(\sigma)-v_{L}(\sigma)$ he receives due to his higher valuation whenever consumption is enforced by the mechanism with positive probability. To prevent type $\theta_{H}$ from misreporting type $\hat{\theta}=\theta_{L}$, the seller needs to pay him at least a rent corresponding to the expected utility increment, as expressed by $R^{x a}$. Because the allocation rule $\tilde{x}^{*}$ in the two type case prescribes consumption exactly when $\sigma$ exceeds the cutoff $\sigma_{L}$, the ex ante information rent $R^{x a}$ corresponds to the area in between the curves $v_{H}(\sigma)$ and $v_{L}(\sigma)$ over the range $\sigma_{L}$ to 1 as depicted in the figure. 


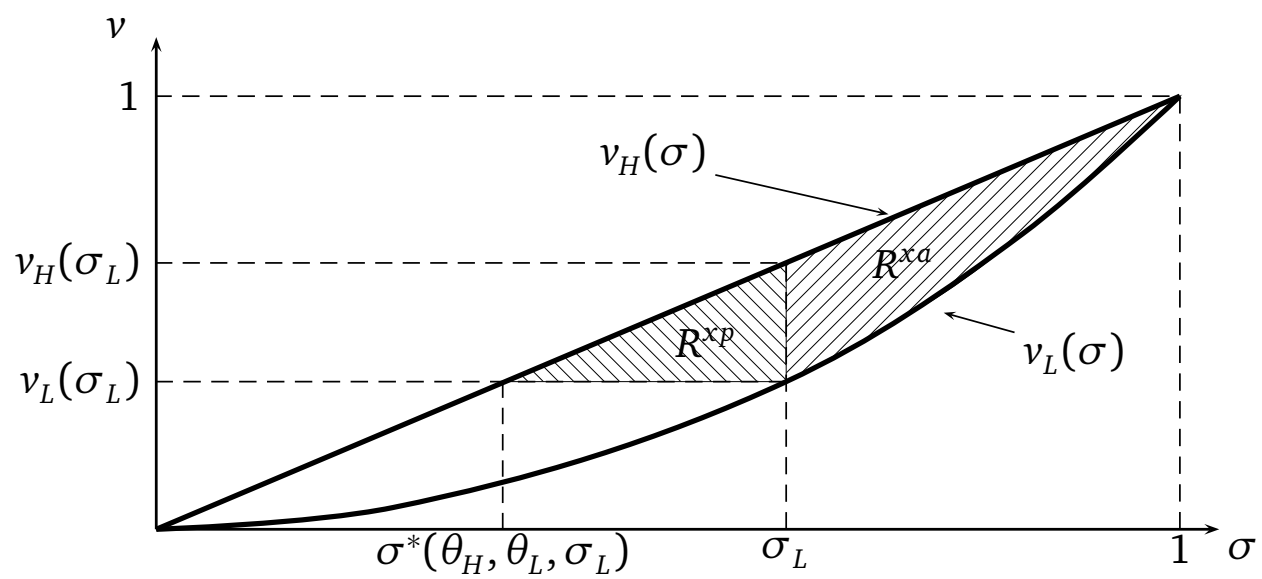

Figure 1: Ex ante vs. ex post information rents.

When the shock is private, the high valuation buyer can increase his utility from a misreport $\hat{\theta}=\theta_{L}$ by combining it with a misreport $\hat{\sigma}$ about the shock that induces his most preferred allocation $x(\hat{\theta}, \hat{\sigma})$ that is available ex post. This additional source of rent is captured by the term $R^{x p}$. The ex post information rent is strictly positive if, conditional on the first report $\hat{\theta}$, the allocation is responsive to the second report $\hat{\sigma}$. Only in this case, there are realizations of $\sigma$ so that the high valuation buyer can exploit the privacy of the shock to induce a better allocation for himself than the allocation that is enforced when $\sigma$ is public. Note that the allocation rule $\tilde{x}^{*}$ with two ex ante types $\theta_{H}$ and $\theta_{L}$ is responsive, as it prescribes consumption only if $\sigma$ exceeds $\sigma_{L}$. As illustrated in Figure 1 this leads to a strictly positive ex post information rent $R^{x p}$. It corresponds to the area between $v_{H}(\sigma)$ and $v_{L}\left(\sigma_{L}\right)$ over the range from $\sigma^{*}$ to $\sigma_{L}$, because exactly for these realization of $\sigma$ a high valuation type would obtain a utility increase of $v_{H}(\sigma)-v_{L}\left(\sigma_{L}\right)$ by reporting $\sigma>\sigma_{L}$ in comparison to reporting $\sigma$ truthfully and ending up with no consumption.

We now use the decomposition to study the relevance of ex post information. We show that ex post information does not give rise to additional rents and is revenue-irrelevant in continuous models, but for discrete models this is true only in the degenerate case that the allocation is not responsive to the second report $\hat{\sigma}$ for the lowest type $\underline{\theta}$.

\subsection{Continuous model}

We first show that for continuous models, the information rents are the same with public and private ex post information, because the marginal ex post information rent, $R^{x p}$ disappears. 


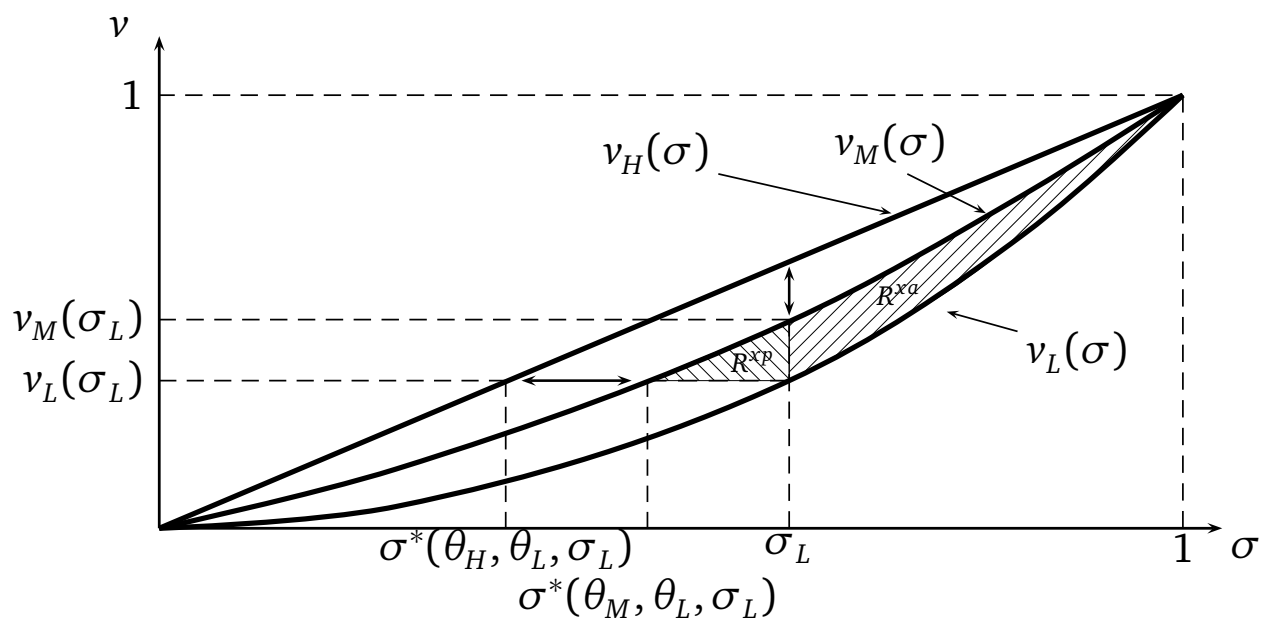

Figure 2: Quadratic reduction of $R^{x p}$ vs. linear reduction of $R^{x a}$.

Lemma 8 Suppose the model is continuous, then for an arbitrary allocation rule $x$, it holds: (a)

$$
\lim _{\delta \rightarrow 0} \frac{R^{x p}(\theta+\delta, \theta, x)}{\delta}=0 .
$$

(b) Moreover, if $x$ is implementable with private shock, then $\tilde{U}$ and $U$ are absolutely continuous and thus differentiable for almost all $\theta \in \Theta$, and whenever the derivatives exist, it holds:

$$
\tilde{U}^{\prime}(\theta)=U^{\prime}(\theta) \geq 0 .
$$

The reason for why the marginal ex post information rent disappears can be intuitively seen as follows. The ex post information rent of a high valuation buyer relative to a low valuation buyer is essentially the product of two factors: the valuation increment and the probability with which the high valuation buyer benefits from misrepresenting the shock $\sigma$ when he pretended to be the low valuation buyer. As the distance between the two ex ante types diminishes, both of these factors go to zero, because the valuation difference diminishes, and, crucially, because the set of realizations $\sigma$ for which a misreport is still beneficial becomes smaller and smaller. Therefore, as the distance between ex ante types goes to zero, the ex post rent converges to zero at a quadratic speed.

In contrast, the ex ante rent converges to zero only at a linear speed, because only the difference in valuations diminishes, but the probability with which consumption is enforced by the mechanism does not diminish as the distance between ex ante types diminishes. 
Figure 2 illustrates this graphically. As a result of introducing an additional intermediate ex ante type $\theta_{M}$, the area $R^{x p}$ shrinks both in the vertical dimension, because the difference in the valuations between type $\theta_{L}$ and the next higher type $\theta_{M}$ becomes smaller, and in the horizontal dimension, because when having announced to be type $\hat{\theta}=\theta_{L}$, type $\theta_{M}$ manipulates the shock $\sigma$ for a smaller range of shocks than type $\theta_{H}$. In contrast, the area $R^{x a}$ shrinks only in the vertical dimension, because the probability with which the contract enforces consumption upon an announcement of type $\hat{\theta}=\theta_{L}$ does not change by the introduction of the intermediate type $\theta_{M}$.

To complete the argument for why ex post information rents are zero in continuous models, notice that since $\tilde{U}^{\prime}(\theta)=U^{\prime}(\theta) \geq 0$, both with public and private shock, the individual rationality constraints (12) and (17) are met if, and only if, they are met for the lowest valuation type $\underline{\theta}$. Therefore, both with public and private shock, the revenue maximizing way for the seller to implement an allocation rule is to have the individual rationality constraint for the lowest type binding: $\tilde{U}(\underline{\theta})=U(\underline{\theta})=0$. Because $\tilde{U}^{\prime}(\theta)=U^{\prime}(\theta)$, and since $\tilde{U}$ and $U$ are absolutely continuous, the buyer's utility is consequently the same with public and private shock, establishing our first main result:

Proposition 1 Suppose the model is continuous. Then it holds:

(a) Any allocation rule $x$ that is implementable with private shock, is implementable without ex post information rents.

(b) (Esö and Szentes $(2007 a, b)$ ) If $\tilde{x}^{*}$ is implementable with private shock, ex post information is revenue-irrelevant.

Part (a) of the proposition says that when the model is continuous, the agent never gets any information rents from his ex post information. This extends Esö and Szentes (2007a, b) who show this result only with respect to the allocation rule that is optimal when the shock is public, as stated in part (b). In particular, if revenue-irrelevance fails in the continuous model, then it fails for implementability reasons, not because the principal has to concede more rents with private than with a public shock.

\subsection{Discrete model}

We now turn to the discrete model and show that, only in rather special cases, ex post information does not increase the agent's rent. Recall that $\left(x^{*}, t^{*}\right)$ is an the optimal mechanism 
with private shock.

Proposition 2 Suppose the model is discrete. Then it holds:

(a) Any allocation rule $x$ that is implementable with private shock is implementable without information rents only if $x(\underline{\theta}, \sigma)$ is constant in $\sigma$ on $(0,1)$.

(b) Ex post information is revenue-irrelevant only if $x^{*}(\underline{\theta}, \sigma)$ is constant in $\sigma$ on $(0,1)$.

To understand the result, note that if $x(\underline{\theta}, \sigma)$ is not constant in $\sigma$, then Lemma 7b implies that the implementation of $x$ with private shock requires that all types larger than $\underline{\theta}$ are paid a strictly positive ex post rent $R^{x p}$ in order to prevent them from claiming to be type $\underline{\theta}$. By Lemma 7a, when the shock is public, the seller can dispense with these extra rents when implementing $x$ and only pay the ex ante rents $R^{x a}$ to the buyer. This applies to any $x$ that is implementable with private shock and, in particular, for $x^{*}$. Hence, the seller can implement the optimal allocation $x^{*}$ with strictly smaller information rents when the shock is public. This means that ex post information is not revenue-irrelevant in discrete models.

For sequential screening models with discrete ex ante types where the so called first order approach works ${ }^{15}$, it is generally known that the optimal sequential mechanism, for any ex ante type $\theta$, prescribes consumption and hence does not display a distortion for the most efficient ex post type $\sigma=1$ (see, e.g., Courty and Li, 2000 or Battaglini, 2005). ${ }^{16}$ In this case, we can strengthen Proposition $2 \mathrm{~b}$ by stating that ex post information is revenue-irrelevant only if the optimal mechanism implements the efficient outcome where the buyer always consumes the good so that there are no distortions.

Finally we point out that our result that ex post information rents are strictly positive does not require a shock that is a continuous random variable. Ex post information rents arise whenever the buyer's optimal report about the shock depends on his true (and not only on his reported) ex ante type. As we have shown, this is always the case when the ex ante information is discrete and the ex post shock is continuous, but, depending on the shock distribution, this is also the case if the shock is discrete. To illustrate, consider a setting with two ex ante types and two possible shocks, favorable $\left(\sigma^{+}\right)$and unfavorable $\left(\sigma^{-}\right)$. We can then distinguish two cases. In the first case, final valuations "overlap". This means that the

\footnotetext{
${ }^{15}$ This means that only the "local" incentive compatibility constraints are binding at the optimum.

${ }^{16}$ There are good reasons to conjecture that this is a general property of optimal sequential mechanisms in any context, but proving this conjecture would go beyond the scope of this paper.
} 
valuation of the buyer with the high ex ante type and the unfavorable shock is smaller than the valuation of the buyer with the low ex ante type and the favorable shock: $v_{H}\left(\sigma^{-}\right) \leq v_{L}\left(\sigma^{+}\right)$. In this case, it can be shown that ex post rents are zero under the optimal selling schedule and therefore ex post information is revenue-irrelevant. ${ }^{17}$ In the other case, final valuations "do not overlap": $v_{H}\left(\sigma^{-}\right)>v_{L}\left(\sigma^{+}\right)$. In this case, when the high ex ante type buyer lies ex ante, he will always report a favorable shock ex post, because irrespective of the true shock his true valuation is even higher than the valuation of the buyer with the low valuation type and the favorable shock and therefore he prefers the terms of trade for this type rather than for the buyer with the low ex ante type and the unfavorable shock. Consequently, when valuations do not overlap, ex post information rents are typically positive. Hence, with discrete shocks, ex post information rents are, in general, also strictly positive.

\section{Disclosure of ex post information}

In this section, we show that our findings have relevant implications for the seller's incentives to disclose ex post information to the buyer. As observed already by Esö and Szentes (2007a, b), when ex post private information is revenue-irrelevant, it is optimal for the seller to fully disclose the shock even if the buyer observes it privately. This is because the seller can extract the full value of the disclosed ex post information as if this information was publicly observable. We now show that full disclosure of the shock is, in fact, not optimal in general. We provide an example of a discrete model in which there exists a partial disclosure policy that allows the seller to attain the same revenue $\tilde{W}^{*}$ as when the shock is public. Hence, under the partial disclosure rule, the ex post private information is revenue-irrelevant, which, as we have seen in Section 4, is not the case under full disclosure.

We reconsider the model with two types from Section 4 and assume that the buyer can only observe the ex ante information $\theta$, but that the extent of the ex post information the buyer observes is controlled by the seller. More specifically, as in Esö and Szentes (2007a, b), we assume that the seller can disclose to the buyer, without observing herself, the realization of any signal that is correlated with the shock $\sigma$. In particular, the seller may adopt a "binary"

\footnotetext{
${ }^{17}$ See Pavan (2007) who presents a general version of this "overlapping" condition (Condition 3, page 23) and shows that this implies the irrelevance result of Esö and Szentes (2007a). Boleslavsky and Said (2013) show for a dynamic screening problem with binomial shock distribution satisfying an overlapping condition that preventing the buyer from misrepresenting his type in a single period is sufficient for truth-telling in any period.
} 
disclosure rule which reveals to the buyer only whether the shock is below or above some cutoff value $\sigma_{0}$. We now consider a specific example in which there is such a partial disclosure rule which allows the seller to attain the revenue $\tilde{W}^{*}$. Let

$$
v_{L}(\sigma)=\sigma^{2}, \quad v_{H}(\sigma)=\sigma .
$$

By Lemma 3, the optimal mechanism with public shock for specification (46) is given by the monotone allocation rule

$$
\tilde{x}_{H}^{*}(\sigma)=1 \quad \forall \sigma \in[0,1], \quad \tilde{x}_{L}^{*}(\sigma)=\left\{\begin{array}{lll}
0 & \text { if } & \sigma<p \\
1 & \text { else, }
\end{array}\right.
$$

and expected payments (by (25) and (26))

$$
\bar{t}_{L}=\int_{p}^{1} v_{L}(\sigma) d \sigma ; \quad \bar{t}_{H}=\int_{0}^{1} v_{H}(\sigma) d \sigma+\int_{p}^{1}\left[v_{L}(\sigma)-v_{H}(\sigma)\right] d \sigma .
$$

Now suppose that the seller commits to a partial disclosure mechanism that reveals to the buyer ex post only whether the shock is below or above the cutoff value $\sigma_{0}=p$. In addition, she offers the buyer the following menu of option contracts:

- By announcing type $\theta_{H}$, the buyer pays the price $\bar{t}_{H}$ and receives the good for sure.

- By announcing the type $\theta_{L}$, the buyer receives the right to buy the good at a price $r_{L}=$ $\bar{t}_{L} /\left(1-\sigma_{0}\right)$ after having learned the additional information.

We claim that this partial disclosure mechanism implements the allocation rule $\tilde{x}^{*}$ and yields the seller the revenue $\tilde{W}^{*}$. More precisely, we show that, under the partial disclosure role, the high valuation buyer does no longer benefit from a lie ex post after having lied initially. In other words, the menu of option contracts does not only implement the allocation rule $\tilde{x}^{*}$ "on the path", after the buyer has announced his type $\theta$ truthfully, but also "off the path", after a lie by the buyer. Note that if this is true, then, on the path, the high valuation buyer consumes the good with probability 1 , inducing the payment $\bar{t}_{H}$ for sure, and the low valuation buyer consumes the good with probability $P\left[\sigma>\sigma_{0}\right]=1-\sigma_{0}$, inducing expected payments $\bar{t}_{L}$. Therefore, not only the allocation rule, but also the expected payments under the partial disclosure mechanism are the same as under the optimal mechanism with publicly observable $\sigma$. This directly implies that the partial disclosure mechanism is ex ante incentive compatible and individually rational and yields the seller the same revenue as the optimal mechanism with public shock. 
To see that the partial disclosure mechanism implements $\tilde{x}^{*}$ on and off the path, observe first that after an announcement $\theta_{H}$, the good is consumed with probability 1 no matter which buyer type made the announcement. Next consider what happens after an announcement $\theta_{L}$, and take first the case that the low valuation buyer has made this announcement. Observe that the exercise price $r_{L}$ exactly matches the expected valuation of the low valuation buyer when he learns that $\sigma$ exceeds $\sigma_{0}$ :

$$
E\left[v_{L}(\sigma) \mid \sigma>\sigma_{0}\right]=\int_{\sigma_{0}}^{1} \frac{v_{L}(\sigma)}{1-\sigma_{0}} d \sigma=\frac{\bar{t}_{L}}{1-\sigma_{0}}=r_{L} .
$$

It is therefore optimal for the low valuation buyer $\theta_{L}$ to exercise the option if, and only if, he learns that $\sigma$ exceeds $\sigma_{0}$.

Next, consider the case that the high valuation buyer announced $\theta_{L}$. Since $v_{H}(\sigma)>v_{L}(\sigma)$ for $\sigma \in(0,1)$, it follows $E\left[v_{H}(\sigma) \mid \sigma>\sigma_{0}\right]>E\left[v_{L}(\sigma) \mid \sigma>\sigma_{0}\right]=r_{L}$, and thus the high valuation buyer exercises the option when he learns that $\sigma>\sigma_{0}$. On the other hand, when he learns that $\sigma<\sigma_{0}$, then using (46), his expected payoff is

$$
E\left[v_{H}(\sigma) \mid \sigma<\sigma_{0}\right]-\bar{t}_{L} /\left(1-\sigma_{0}\right)=\sigma_{0} / 2-\left(1-\sigma_{0}^{3}\right) /\left(3-3 \sigma_{0}\right)=-\left(2 \sigma_{0}^{2}-\sigma_{0}+2\right) / 6<0 .(50)
$$

The inequality follows because the expression attains a maximum of $-5 / 16$ at $\sigma_{0}=1 / 4$.

This shows that the partial disclosure mechanism implements $\tilde{x}^{*}$ on and off the path, and, as a result, the seller obtains the expected revenue $\tilde{W}^{*}$. As shown in Section 4, this is more than what the seller can obtain under full disclosure, and therefore partial disclosure is strictly optimal.

Intuitively, partial disclosure eliminates the ex post information rent, because it affects the way how the buyer uses his ex post information. Even though the high valuation buyer, after having announced $\theta_{L}$, is still free to misrepresent the shock, the disclosure policy is designed so that it is no longer optimal for the buyer to do so, but rather to choose the same allocation that would be enforced when the shock were public. In this case, ex post information does not yield additional rents.

That there is a disclosure rule that implements the same outcome as if the shock was public, is a special feature of our example. On a more general note, restricting the ex post information available to the buyer has two countervailing effects. On the one hand, it reduces ex post information rents, as it diminishes the value from misrepresenting ex post information 
off the path. On the other hand, it also reduces the set of allocations that can be implemented on the path. We conjecture that, unlike in our example, there is in general no disclosure rule that fully resolves this trade-off and permits the seller to attain $\tilde{W}^{*}$.

The effect that revealing less information reduces information rents and may therefore be beneficial to the seller, is reminiscent of a general insight in mechanism design that "revealing more information to players makes it harder to prevent them from finding ways to gain by lying" (Myerson 1991, p.297) so that one should reveal "to each player only the minimum information needed to guide his action" (Myerson 1991, p.260). Indeed in our example, revealing only whether $\sigma$ is larger or smaller than $\sigma_{0}=p$ is the minimum amount of information revelation that is needed to implement the allocation rule. Disclosing more information only makes it more difficult to satisfy incentive compatibility, leading to higher implementation costs in the form of positive ex post information rents.

We close this section by clarifying the relationship between our partial disclosure result and a recent partial disclosure result by Li and Shi (2013). Li and Shi (2013) consider a seller who can disclose, without observing herself, information not about the shock but about the final valuation $v$ and show that in this case, partial disclosure might be optimal, even in the continuous setting of Esö and Szentes (2007a, b). Effectively, when the seller can disclose information about the final valuation, she can choose from a larger set of disclosure policies than when she can disclose information only about the shock. Imagine that in our example, the seller wanted to disclose to the buyer whether his valuation $v$ is larger than $1 / 4$. This information cannot be disclosed by disclosure of the shock alone, because the seller would need to disclose to the high valuation buyer whether $\nu_{H}(\sigma)=\sigma>1 / 4$, i.e. whether the shock is larger than $1 / 4$, but to the low valuation buyer the seller would need to disclose whether $v_{L}(\sigma)=\sigma^{2}>1 / 4$, i.e. whether the shock is larger than $1 / 2$. For this larger class of disclosure policies, Li and Shi (2013) show that not only full disclosure of $v$ may be suboptimal but also that there can be partial disclosure policies that allow the seller to obtain a higher revenue than in the benchmark of Esö and Szentes (2007a, b).

\section{Conclusion}

In this paper we show that, in general, a principal has to pay information rents for inducing an agent to reveal his private ex post information in excess of the rents that she needs to 
pay for eliciting the agent's private ex ante information. We interpret these additional rents as ex post information rents. The principal has to pay such rents, because in their absence the agent would benefit from coordinating his ex ante and ex post lies. The agent's potential benefits from such coordinated lying are strictly positive when the ex ante private information is discrete and the ex post private information is continuous. In such a model, the classical trade-off between rents and efficiency is more subtle, because the rents consist of both ex ante and ex post information rents. 


\section{Appendix}

Proof of Lemma 1: (a) The claim follows directly from the assumption that $F(v \mid \theta)$ first order stochastically dominates $F(v \mid \theta)$ for $\theta>\hat{\theta}$.

(b) The claim follows from the non-shifting support assumption, and because $F(v \mid \theta)$ is strictly increasing and continuous in $v$ for all $\theta \in \Theta$.

(c) Existence of $\partial v(\theta, \sigma) / \partial \sigma$ follows because $F$ has a density $f$. In particular, $\partial v(\theta, \sigma) / \partial \sigma=$ $1 / f(v(\theta, \sigma) \mid \theta)$ is strictly positive and bounded because $f(v, \theta)$ is strictly positive and bounded away from zero.

(d) Finally, if the model is continuous, then $\partial F(v \mid \theta) / \partial \theta$ exists and, because in addition $f(v \mid \theta)>0$, we can apply the implicit function theorem to obtain $\partial v(\theta, \sigma) / \partial \theta$ by differentiating the identity $F(v(\theta, \sigma) \mid \theta)=\sigma$ with respect to $\theta$ :

$$
\frac{\partial F(v \mid \theta)}{\partial v} \frac{\partial v(\theta, \sigma)}{\partial \theta}+\frac{\partial F(v \mid \theta)}{\partial \theta}=0 \Leftrightarrow \frac{\partial v(\theta, \sigma)}{\partial \theta}=-\frac{\partial F(v \mid \theta)}{\partial \theta} \frac{1}{f(v \mid \theta)} .
$$

Boundedness of $\partial v(\theta, \sigma) / \partial \theta$ follows because $\partial F(v \mid \theta) / \partial \theta$ is bounded and $f(v \mid \theta)$ is bounded away from zero.

Proof of Lemma 2: Adding up $\left(\mathrm{IC}_{H}\right)$ and $\left(\mathrm{IC}_{L}\right)$ and re-arranging yields:

$$
\int_{0}^{1}\left[v_{H}(\sigma)-v_{L}(\sigma)\right]\left[\tilde{x}_{H}(\sigma)-\tilde{x}_{L}(\sigma)\right] d \sigma \geq 0
$$

Moreover, $\left(\mathrm{IC}_{H}\right)$ and $\left(\mathrm{IR}_{L}\right)$ imply $\left(\mathrm{IR}_{H}\right)$ because of (20). Therefore, $\left(\mathrm{IR}_{H}\right)$ can be ignored, and it follows that at the optimum, $\left(\mathrm{IR}_{L}\right)$ and $\left(\mathrm{IC}_{H}\right)$ are binding, because otherwise, without affecting any other constraint, the expected transfers to type $\theta_{L}$ could be reduced until $\left(\mathrm{IR}_{L}\right)$ binds, and the expected transfers to type $\theta_{H}$ could be reduced until $\left(\mathrm{IC}_{H}\right)$ binds. Since $\left(\mathrm{IC}_{H}\right)$ is binding, (52) implies

$$
\int_{0}^{1} \tilde{t}_{L}(\sigma)-\tilde{t}_{H}(\sigma) d \sigma=\int_{0}^{1} v_{H}(\sigma)\left[\tilde{x}_{L}(\sigma)-\tilde{x}_{H}(\sigma)\right] d \sigma \leq \int_{0}^{1} v_{L}(\sigma)\left[\tilde{x}_{L}(\sigma)-\tilde{x}_{H}(\sigma)\right],
$$

but this inequality is the same as $\left(\mathrm{IC}_{L}\right)$. Hence, also $\left(\mathrm{IC}_{L}\right)$ can be ignored, and the claim follows.

Q.E.D.

Proof of Lemma 3 Follows directly from the main text.

Q.E.D.

Proof of Lemma 4 Follows directly from the main text.

Proof of Lemma 5 Standard and therefore omitted. 
Proof of Lemma 6 By Lemma $1 \mathrm{~b}$, there is a unique $\sigma^{*}$ so that $v\left(\theta, \sigma^{*}\right)=v\left(\hat{\theta}, \sigma^{*}\right)$. If type $\theta$ has reported type $\hat{\theta}$ and observed $\sigma$, he chooses $\hat{\sigma}$ to maximize

$$
v(\theta, \sigma) x(\hat{\theta}, \hat{\sigma})-t(\hat{\theta}, \hat{\sigma})=v\left(\hat{\theta}, \sigma^{*}\right) x(\hat{\theta}, \hat{\sigma})-t(\hat{\theta}, \hat{\sigma})
$$

By ex post incentive compatibility for type $\hat{\theta}$, the report $\hat{\sigma}=\sigma^{*}$ maximizes the right hand side of (54). Thus, $\sigma^{*}$ is an optimal report in the second stage when type $\theta$ has reported type $\hat{\theta}$ and observed $\sigma$.

To see (b), note that because of (a), we have

$$
u\left(\hat{\theta}, \sigma^{*} ; \theta, \sigma\right)=v(\theta, \sigma) x\left(\hat{\theta}, \sigma^{*}\right)-t\left(\hat{\theta}, \sigma^{*}\right)=v\left(\hat{\theta}, \sigma^{*}\right) x\left(\hat{\theta}, \sigma^{*}\right)-t\left(\hat{\theta}, \sigma^{*}\right)=u\left(\hat{\theta}, \sigma^{*}\right)
$$

This shows the result.

Q.E.D.

Proof of Lemma 7 (a) Immediate from (15) and (16).

(b) By Lemma 5, it is sufficient to show that ex ante incentive compatibility (11) is equivalent to (43). Indeed, by ex post incentive compatibility we have that $U(\hat{\theta})=\int_{0}^{1} u(\hat{\theta}, \sigma) d \sigma$. Hence, ex ante incentive compatibility (11) is equivalent to

$$
\begin{aligned}
U(\theta)-U(\hat{\theta}) & \geq \int_{0}^{1} \max _{\hat{\sigma}} u(\hat{\theta}, \hat{\sigma} ; \theta, \sigma) d \sigma-\int_{0}^{1} u(\hat{\theta}, \sigma) d \sigma \\
& =\int_{0}^{1} u\left(\hat{\theta}, \sigma^{*}\right)-u(\hat{\theta}, \sigma) d \sigma \\
& =\int_{0}^{1} \int_{\sigma}^{\sigma^{*}} \frac{\partial u(\hat{\theta}, c)}{\partial \sigma} d c d \sigma \\
& =\int_{0}^{1} \int_{\sigma}^{\sigma^{*}} \frac{\partial v(\hat{\theta}, c)}{\partial \sigma} x(\hat{\theta}, c) d c d \sigma,
\end{aligned}
$$

where the first equality follows from Lemma $6 \mathrm{~b}$ and the final equality follows from Lemma 5.

Moreover, by Lemma 6a, we have that

$$
\begin{aligned}
R^{x a}(\theta, \hat{\theta}, x) & =\int_{0}^{1}[v(\theta, \sigma)-v(\hat{\theta}, \sigma)] x(\hat{\theta}, \sigma) d \sigma \\
& =\int_{0}^{1}\left[v\left(\hat{\theta}, \sigma^{*}\right)-v(\hat{\theta}, \sigma)\right] x(\hat{\theta}, \sigma) d \sigma \\
& =\int_{0}^{1} \int_{\sigma}^{\sigma^{*}} \frac{\partial v(\hat{\theta}, c)}{\partial \sigma} x(\hat{\theta}, \sigma) d c d \sigma
\end{aligned}
$$

Adding the last term to $R^{x p}(\theta, \hat{\theta}, x)$ yields expression (59), and hence, (43) holds. 
Moreover, to see that $R^{x p}(\theta, \hat{\theta}, x)$ is positive, observe that $\sigma \gtrless \sigma^{*}(\theta, \hat{\theta}, \sigma) \Leftrightarrow \theta \lessgtr \hat{\theta}$ for all $\sigma \in(0,1)$ by (3) and Lemma 6a. Hence, since $\partial v / \partial \sigma$ is positive by Lemma $1 \mathrm{c}$, and $x(\hat{\theta}, \sigma)$ is non-decreasing in $\sigma$ according to Lemma $5, R^{x p}$ is positive.

Finally, suppose that $x(\hat{\theta}, \sigma)$ is not constant in $\sigma$ on $(0,1)$, and consider the case $\theta>\hat{\theta}$. (The case $\theta<\hat{\theta}$ can be treated analogously.) Then, because $x(\hat{\theta}, \sigma)$ is non-decreasing in $\sigma$ according to Lemma 5 , there exist $0<\sigma_{1}<\sigma_{2}^{\prime}<1$ such that $x\left(\hat{\theta}, \sigma_{1}\right)<x\left(\hat{\theta}, \sigma_{2}^{\prime}\right)$ and $x(\hat{\theta}, \sigma)$ is not constant on the open interval $\left(\sigma_{1}, \sigma_{2}^{\prime}\right)$. Define

$$
\sigma_{2}=\inf \left\{\sigma \mid x(\hat{\theta}, \sigma)=x\left(\hat{\theta}, \sigma_{2}^{\prime}\right)\right\} .
$$

Because $x(\hat{\theta}, \sigma)$ is not constant on the open interval $\left(\sigma_{1}, \sigma_{2}^{\prime}\right)$, we have that $\sigma_{1}<\sigma_{2}$. Let

$$
\Delta \sigma=\min _{\sigma \in\left[\sigma_{1}, \sigma_{2}\right]} \sigma^{*}(\theta, \hat{\theta}, \sigma)-\sigma .
$$

Note that $\Delta \sigma$ exists and, since $\theta>\hat{\theta}$ and $0<\sigma_{1}<\sigma_{2}<1$, we have $\Delta \sigma>0$.

Now let

$$
\sigma_{0}=\sigma_{2}-1 / 2 \cdot \Delta \sigma
$$

Then, by definition of $\sigma_{2}$, we have for all $\sigma \in\left[\sigma_{0}, \sigma_{2}\right)$ that $\sigma<\sigma_{2}$ and $\sigma+\Delta \sigma>\sigma_{2}$ so that

$$
\int_{\sigma}^{\sigma+\Delta \sigma}\{x(\hat{\theta}, c)-x(\hat{\theta}, \sigma)\} d c>0 .
$$

Therefore,

$$
\int_{\sigma_{0}}^{\sigma_{2}} \int_{\sigma}^{\sigma+\Delta \sigma}\{x(\hat{\theta}, c)-x(\hat{\theta}, \sigma)\} d c d \sigma>0
$$

Moreover, let

$$
k=\min _{c \in\left[\sigma_{0}, \sigma_{2}\right]} \frac{\partial v(\hat{\theta}, c)}{\partial \sigma}>0,
$$

which is strictly positive by Lemma 1c.

Applying these properties together with $x(\hat{\theta}, \sigma)$ being non-decreasing in $\sigma$, we can infer:

$$
\begin{aligned}
R^{x p}(\theta, \hat{\theta}, x) & =\int_{0}^{1} \int_{\sigma}^{\sigma^{*}(\theta, \hat{\theta}, \sigma)} \frac{\partial v(\hat{\theta}, c)}{\partial \sigma}[x(\hat{\theta}, c)-x(\hat{\theta}, \sigma)] d c d \sigma \\
& \geq \int_{\sigma_{0}}^{\sigma_{2}} \int_{\sigma}^{\sigma^{*}(\theta, \hat{\theta}, \sigma)} \frac{\partial v(\hat{\theta}, c)}{\partial \sigma}[x(\hat{\theta}, c)-x(\hat{\theta}, \sigma)] d c d \sigma \\
& \geq k \int_{\sigma_{0}}^{\sigma_{2}} \int_{\sigma}^{\sigma+\Delta \sigma}[x(\hat{\theta}, c)-x(\hat{\theta}, \sigma)] d c d \sigma>0 .
\end{aligned}
$$


where the final inequality follows from (67). This shows that $R^{x p}(\theta, \hat{\theta}, x)>0$ if $x(\hat{\theta}, \sigma)$ is not constant in $\sigma$ on $(0,1)$ and completes the proof.

Q.E.D.

Proof of Lemma 8 (a) Note that $\sigma^{*}(\theta, \theta, \sigma)=\sigma$. Hence,

$$
\lim _{\delta \rightarrow 0} \frac{R^{x p}(\theta+\delta, \theta, x)}{\delta}=\int_{0}^{1}\left[\lim _{\delta \rightarrow 0} \frac{1}{\delta} \int_{\sigma^{*}(\theta, \theta, \sigma)}^{\sigma^{*}(\theta+\delta, \theta, \sigma)} \frac{\partial v(\theta, c)}{\partial \sigma}[x(\theta, c)-x(\theta, \sigma)] d c\right] d \sigma,
$$

because we can interchange integration and taking the limit since all functions are bounded. Now observe that because the model is continuous, we have, by $(6)$, that $v(\theta, \sigma)$ is differentiable in $\theta$, and thus Lemma 6 a, implies that $\sigma^{*}(\theta, \hat{\theta}, \sigma)$ is differentiable in $\theta$. Hence, by Leibniz' rule, the term in the square bracket in (72) is equal to

$$
\left.\frac{\partial v\left(\theta, \sigma^{*}(\theta+\delta, \theta, \sigma)\right)}{\partial \sigma}\left[x\left(\theta, \sigma^{*}(\theta+\delta, \theta, \sigma)\right)-x(\theta, \sigma)\right] \cdot \frac{\partial \sigma^{*}(\theta+\delta, \theta, \sigma)}{\partial \theta}\right|_{\delta=0}=0
$$

because $\left.x\left(\theta, \sigma^{*}(\theta+\delta, \theta, \sigma)\right)\right|_{\delta=0}=x\left(\theta, \sigma^{*}(\theta, \theta, \sigma)\right)=x(\theta, \sigma)$ so that the difference in the squared brackets in (73) is zero, and this shows part (a).

(b) To see absolute continuity, note first that for an arbitrary function $\phi(\hat{z} ; z)$ for which $\max _{\hat{z}} \phi(\hat{z} ; z)$ is well-defined, it holds for all $z_{1}, z_{2}$ :

$$
\left|\max _{\hat{z}} \phi\left(\hat{z} ; z_{1}\right)-\max _{\hat{z}} \phi\left(\hat{z} ; z_{2}\right)\right| \leq \max _{\hat{z}}\left|\phi\left(\hat{z} ; z_{1}\right)-\phi\left(\hat{z} ; z_{2}\right)\right| .
$$

As absolute continuity is implied by Lipschitz continuity, it is sufficient to show that $U$ and $\tilde{U}$ are Lipschitz continuous. To see the Lipschitz continuity of $\tilde{U}$, observe that incentive compatibility, (16), implies $\tilde{U}(\theta)=\max _{\hat{\theta}} \tilde{U}(\hat{\theta} ; \theta)$, and hence by (74),

$$
\begin{aligned}
\left|\tilde{U}\left(\theta_{1}\right)-\tilde{U}\left(\theta_{2}\right)\right| & \leq \max _{\hat{\theta}}\left|\tilde{U}\left(\hat{\theta} ; \theta_{1}\right)-\tilde{U}\left(\hat{\theta} ; \theta_{2}\right)\right| \\
& =\max _{\hat{\theta}}\left|\int_{0}^{1}\left\{v\left(\theta_{1}, \sigma\right)-v\left(\theta_{2}, \sigma\right)\right\} x(\hat{\theta}, \sigma) d \sigma\right| \\
& \leq \int_{0}^{1}\left|v\left(\theta_{1}, \sigma\right)-v\left(\theta_{2}, \sigma\right)\right| d \sigma \\
& \leq \int_{0}^{1} \max _{\tilde{\theta}}\left|\frac{\partial v(\tilde{\theta}, \sigma)}{\partial \theta}\left\{\theta_{1}-\theta_{2}\right\}\right| d \sigma \\
& \leq \max _{\tilde{\theta}, \sigma}\left|\frac{\partial v(\tilde{\theta}, \sigma)}{\partial \theta}\right|\left|\theta_{1}-\theta_{2}\right|,
\end{aligned}
$$


where the second line follows from the definition of $\tilde{U}$ in (15), the third line from $x(\hat{\theta}, \sigma) \leq 1$, and the final lines from standard estimations and the fact that $\partial v / \partial \theta$ is bounded by Lemma 1d. This proves that $\tilde{U}$ is Lipschitz continuous with Lipschitz constant $\max _{\tilde{\theta}, \sigma}|\partial v(\tilde{\theta}, \sigma) / \partial \theta|$. To see Lipschitz continuity of $U$, observe first that (74) implies for all $\hat{\theta}, \theta_{1}, \theta_{2}, \sigma$ :

$$
\left|\max _{\hat{\sigma}} u\left(\hat{\theta}, \hat{\sigma} ; \theta_{1}, \sigma\right)-\max _{\hat{\sigma}} u\left(\hat{\theta}, \hat{\sigma} ; \theta_{2}, \sigma\right)\right| \leq \max _{\hat{\sigma}}\left|u\left(\hat{\theta}, \hat{\sigma} ; \theta_{1}, \sigma\right)-u\left(\hat{\theta}, \hat{\sigma} ; \theta_{2}, \sigma\right)\right| .
$$

By incentive compatibility, (11), we have $U(\theta)=\max _{\hat{\theta}} U(\hat{\theta} ; \theta)$, and, by definition, $U(\hat{\theta} ; \theta)=$ $\int_{0}^{1} \max _{\hat{\sigma}} u(\hat{\theta}, \hat{\sigma} ; \theta, \sigma)$. Hence, applying again (74) and then (80), we obtain

$$
\begin{aligned}
\left|U\left(\theta_{1}\right)-U\left(\theta_{2}\right)\right| & \leq \max _{\hat{\theta}}\left|U\left(\hat{\theta} ; \theta_{1}\right)-U\left(\hat{\theta} ; \theta_{2}\right)\right| \\
& \leq \max _{\hat{\theta}} \int_{0}^{1} \max _{\hat{\sigma}}\left|u\left(\hat{\theta}, \hat{\sigma} ; \theta_{1}, \sigma\right)-u\left(\hat{\theta}, \hat{\sigma} ; \theta_{2}, \sigma\right)\right| d \sigma \\
& =\max _{\hat{\theta}} \int_{0}^{1} \max _{\hat{\sigma}}\left|\left\{v\left(\theta_{1}, \sigma\right)-v\left(\theta_{2}, \sigma\right)\right\} x(\hat{\theta}, \hat{\sigma})\right| d \sigma \\
& \leq \int_{0}^{1}\left|v\left(\theta_{1}, \sigma\right)-v\left(\theta_{2}, \sigma\right)\right| d \sigma,
\end{aligned}
$$

where the third line follows from the definition of $u$ in (8), and the last line from the fact that $x(\hat{\theta}, \hat{\sigma}) \leq 1$. The same steps as in the previous paragraph now imply that $U$ is Lipschitz continuous.

Since $U$ and $\tilde{U}$ are Lipschitz, they are absolutely continuous, and thus differentiable almost everywhere. Consider $\theta \in \Theta$ at which $\tilde{U}^{\prime}$ exists. Then, by Lemma 7a:

$$
\tilde{U}^{\prime}(\theta)=\lim _{\delta \rightarrow 0} \frac{\tilde{U}(\theta+\delta)-\tilde{U}(\theta)}{\delta} \geq \lim _{\delta \rightarrow 0} \frac{R^{x a}(\theta+\delta, \theta, x)}{\delta}=\int_{0}^{1} \frac{\partial v(\theta, \sigma)}{\partial \theta} x(\theta, \sigma) d \sigma,
$$

and

$$
\tilde{U}^{\prime}(\theta)=\lim _{\delta \rightarrow 0} \frac{\tilde{U}(s)-\tilde{U}(\theta-\delta)}{\delta} \leq \lim _{\delta \rightarrow 0}-\frac{R^{x a}(\theta-\delta, \theta, x)}{\delta}=\int_{0}^{1} \frac{\partial v(\theta, \sigma)}{\partial \theta} x(\theta, \sigma) d \sigma,
$$

where the limits on the right hand side exist, because $\partial v(\theta, \sigma) / \partial \theta$ exists and is bounded. The two inequalities imply that

$$
\tilde{U}^{\prime}(\theta)=\int_{0}^{1} \frac{\partial v(\theta, \sigma)}{\partial \theta} x(\theta, \sigma) d \sigma .
$$

With analogous reasoning, we can deduce by Lemma 7b that for all $\theta \in \Theta$ at which $U^{\prime}$ exists, 
we have

$$
\begin{aligned}
U^{\prime}(\theta) & =\lim _{\delta \rightarrow 0} 1 / \delta \cdot R^{x a}(\theta+\delta, \theta, x)+\lim _{\delta \rightarrow 0} 1 / \delta \cdot R^{x p}(\theta+\delta, \theta, x) \\
& =\int_{0}^{1} \frac{\partial v(\theta, \sigma)}{\partial \theta} x(\theta, \sigma) d \sigma+0,
\end{aligned}
$$

where the final equality follows from part (a). Hence we have shown that $\tilde{U}^{\prime}(\theta)=U^{\prime}(\theta)$ for all $\theta \in \Theta$ at which the derivatives exist. Moreover, since $\partial v / \partial \theta \geq 0$ by Lemma 1a, the derivatives are positive. This completes the proof of part (b).

Proof of Proposition 1 Follows directly from the main text.

Q.E.D.

Proof of Proposition 2 Note that (b) directly follows from (a) with $x=x^{*}$. Hence, we only show (a). If $x$ is implementable with private shock, then there exists a transfer schedule $t$ such that $(x, t)$ is feasible with private shock. Denote by $\hat{t}$ a solution to (14), that is, $(x, \hat{t})$ is feasible and

$$
W(x)=\int_{\Theta} \int_{0}^{1} \hat{t}(\theta, \sigma) d \sigma d P(\theta) .
$$

We now show that if $x(\underline{\theta}, \sigma)$ is not constant in $\sigma$ on $(0,1)$, then $W(x)<\tilde{W}(x)$, which implies that $x$ is not implementable without ex post information rents. We prove this result by constructing an alternative set of transfers $\tilde{t}$ such that $(x, \tilde{t})$ is feasible with public shock and satisfies

$$
W(x)<\int_{\Theta} \int_{0}^{1} \tilde{t}(\theta, \sigma) d \sigma d P(\theta) .
$$

Indeed, define

$$
R \equiv \min _{\theta \neq \underline{\theta}} R^{x p}(\theta, \underline{\theta}, x)
$$

For the discrete model, $\Theta$ is finite and, therefore, the minimum exists. Since $x(\underline{\theta}, \sigma)$ is not constant in $\sigma$ on $(0,1)$, Lemma $7 \mathrm{~b}$, implies that $R>0$. Define now the alternative payment scheme $\tilde{t}$ by

$$
\tilde{t}(\theta, \sigma)= \begin{cases}\hat{t}(\underline{\theta}, \sigma) & \text { if } \theta=\underline{\theta} \\ \hat{t}(\theta, \sigma)+R & \text { otherwise. }\end{cases}
$$

Clearly, $\tilde{t}$ satisfies (91). In order to show that $(x, \tilde{t})$ is also feasible with public shock, let $\tilde{U}(\theta)$ be the expected utility of buyer type $\theta$ from the mechanism $(x, \tilde{t})$ when he truthfully reports 
his type $\theta$, and $\sigma$ is publicly observable, as defined after equation (15). Likewise, let $U(\theta)$ be the expected utility of buyer type $\theta$ from the mechanism $(x, \hat{t})$ when he truthfully reports his type $\theta$, and $\sigma$ is privately observable, as defined after equation (10). By construction of the payments, we have

$$
\tilde{U}(\theta)=U(\theta)-R \quad \forall s \neq \underline{\theta}, \quad \tilde{U}(\underline{\theta})=U(\underline{\theta}) .
$$

We now show that $(x, \tilde{t})$ is incentive compatible with public shock. Indeed, since $(x, \hat{t})$ is incentive compatible with private shock, Lemma $7 \mathrm{~b}$, implies for all $\theta \neq \underline{\theta}, \hat{\theta} \neq \underline{\theta}$,

$$
\tilde{U}(\theta)-\tilde{U}(\hat{\theta})=U(\theta)-U(\hat{\theta}) \geq R^{x a}(\theta, \hat{\theta}, \tilde{x})+R^{x p}(\theta, \hat{\theta}, \tilde{x}) \geq R^{x a}(\theta, \hat{\theta}, \tilde{x}),
$$

and for all $\theta \neq \underline{\theta}$,

$$
\tilde{U}(\theta)-\tilde{U}(\underline{\theta})=U(\theta)-U(\underline{\theta})-R \geq R^{x a}(\theta, \underline{\theta}, \tilde{x})+R^{x p}(\theta, \underline{\theta}, \tilde{x})-R \geq R^{x a}(\theta, \underline{\theta}, \tilde{x}),
$$

and for all $\hat{\theta} \neq \underline{\theta}$,

$$
\tilde{U}(\underline{\theta})-\tilde{U}(\hat{\theta})=U(\underline{\theta})-U(\hat{\theta})+R \geq R^{x a}(\underline{\theta}, \hat{\theta}, \tilde{x})+R^{x p}(\underline{\theta}, \hat{\theta}, \tilde{x})+R \geq R^{x a}(\underline{\theta}, \hat{\theta}, \tilde{x}) .
$$

Hence, $\tilde{U}$ satisfies the inequalities in Lemma $7 \mathrm{a}$, and thus $(x, \tilde{t})$ is incentive compatible with public shock.

It remains to show that $(x, \tilde{t})$ is individually rational with public shock. Indeed, since $(x, \hat{t})$ is individually rational with private shock, we have $\tilde{U}(\underline{\theta})=U(\underline{\theta}) \geq 0$. Since we established in the proof of Lemma $8 \mathrm{~b}$ that $\tilde{U}(\theta)$ is increasing in $\theta$ whenever the mechanism is incentive compatible with public shock, we have $\tilde{U}(\theta) \geq \tilde{U}(\underline{\theta}) \geq 0$ for all $\theta \in \Theta$ so that $(x, \tilde{t})$ is individually rational. And this completes the proof.

Q.E.D.

\section{References}

Baron, D. and D. Besanko (1984). "Regulation and Information in a Continuing Relationship." Information Economics and Policy 1, 267-302.

Baron, D. and R. Myerson (1982). "Regulating a Monopolist with Unknown Costs." Econometrica 50, 911-930.

Battaglini, M. (2005). "Long-Term Contracting with Markovian Consumers." American Economic Review 95, 637-658. 
Bergemann, D. and A. Wambach (2013). "Sequential Information Disclosure in Auctions." mimeo Yale University.

Boleslavsky, R. and M. Said (2013). "Progressive Screening: Long-Term Contracting with a Privately Known Stochastic Process." Review of Economic Studies 80, 1-34.

Courty, P. and H. Li (2000). "Sequential Screening." Review of Economic Studies 67, 697-717.

Deb, R. and M. Said (2013). "Dynamic Screening with Limited Commitment." mimeo University of Toronto.

Esö, P. and B. Szentes (2007a). "The Price of Advise." RAND Journal of Economics 38, 863880.

Esö, P. and B. Szentes (2007b). "Optimal Information Disclosure in Auctions and the Handicap Auction." Review of Economic Studies 74, 705-731.

Esö, P. and B. Szentes (2013). "Dynamic Contracting: An Irrelevance Result." mimeo London School of Economics.

Harris, M. and A. Raviv (1978). "Some Results on Incentive Contracts with Applications to Education and Employment, Health Insurance, and Law Enforcement." American Economic Review 68, 20-30.

Hoffmann, F. and R. Inderst (2011). "Pre-sale information." Journal of Economic Theory 146, 2033-2055.

Krähmer, D. and R. Strausz (2011). "Optimal Procurement Contracts with Pre-Project Planning." Review of Economic Studies 78, 1015-1041.

Laffont, J.-J. and J. Tirole (1986). "Using Cost Observation to Regulate Firms." Journal of Political Economy 94, 614-641.

Li, H. and Shi, X. (2013). "Discriminatory Information Disclosure." mimeo University of British Columbia.

Myerson, R. (1986). "Multistage Games with Communication." Econometrica 54, 323-58.

Myerson, R. (1991). Game Theory: Analysis of Conflict, Harvard University Press, Cambridge, Massachusetts. 
Nocke, V., Peitz, M., and F. Rosar (2011). "Advance-Purchase Discounts as a Price Discrimination Device." Journal of Economic Theory 146, 141-162.

Pavan, A. (2007). "Long-Term Contracting in a Changing World." mimeo Northwestern University.

Pavan, A., I. Segal, and J. Toikka (2013). "Dynamic Mechanism Design: A Myersonian Approach" Econometrica, forthcoming. 


\section{SFB 649 Discussion Paper Series 2014}

For a complete list of Discussion Papers published by the SFB 649, please visit http://sfb649.wiwi.hu-berlin.de.

001 "Principal Component Analysis in an Asymmetric Norm" by Ngoc Mai Tran, Maria Osipenko and Wolfgang Karl Härdle, January 2014.

002 "A Simultaneous Confidence Corridor for Varying Coefficient Regression with Sparse Functional Data" by Lijie Gu, Li Wang, Wolfgang Karl Härdle and Lijian Yang, January 2014.

003 "An Extended Single Index Model with Missing Response at Random" by Qihua Wang, Tao Zhang, Wolfgang Karl Härdle, January 2014.

004 "Structural Vector Autoregressive Analysis in a Data Rich Environment: A Survey" by Helmut Lütkepohl, January 2014.

005 "Functional stable limit theorems for efficient spectral covolatility estimators" by Randolf Altmeyer and Markus Bibinger, January 2014.

006 "A consistent two-factor model for pricing temperature derivatives" by Andreas Groll, Brenda López-Cabrera and Thilo Meyer-Brandis, January 2014.

007 "Confidence Bands for Impulse Responses: Bonferroni versus Wald" by Helmut Lütkepohl, Anna Staszewska-Bystrova and Peter Winker, January 2014.

008 "Simultaneous Confidence Corridors and Variable Selection for Generalized Additive Models" by Shuzhuan Zheng, Rong Liu, Lijian Yang and Wolfgang Karl Härdle, January 2014.

009 "Structural Vector Autoregressions: Checking Identifying Long-run Restrictions via Heteroskedasticity" by Helmut Lütkepohl and Anton Velinov, January 2014.

010 "Efficient Iterative Maximum Likelihood Estimation of HighParameterized Time Series Models" by Nikolaus Hautsch, Ostap Okhrin and Alexander Ristig, January 2014.

011 "Fiscal Devaluation in a Monetary Union" by Philipp Engler, Giovanni Ganelli, Juha Tervala and Simon Voigts, January 2014.

012 "Nonparametric Estimates for Conditional Quantiles of Time Series" by Jürgen Franke, Peter Mwita and Weining Wang, January 2014.

013 "Product Market Deregulation and Employment Outcomes: Evidence from the German Retail Sector" by Charlotte Senftleben-König, January 2014.

014 "Estimation procedures for exchangeable Marshall copulas with hydrological application" by Fabrizio Durante and Ostap Okhrin, January 2014.

015 "Ladislaus von Bortkiewicz - statistician, economist, and a European intellectual" by Wolfgang Karl Härdle and Annette B. Vogt, February 2014.

016 "An Application of Principal Component Analysis on Multivariate TimeStationary Spatio-Temporal Data" by Stephan Stahlschmidt, Wolfgang Karl Härdle and Helmut Thome, February 2014.

017 "The composition of government spending and the multiplier at the Zero Lower Bound" by Julien Albertini, Arthur Poirier and Jordan RoulleauPasdeloup, February 2014.

018 "Interacting Product and Labor Market Regulation and the Impact of Immigration on Native Wages" by Susanne Prantl and Alexandra SpitzOener, February 2014.

\section{SFB 649, Spandauer Straße 1, D-10178 Berlin http://sfb649.wiwi.hu-berlin.de}




\title{
SFB 649 Discussion Paper Series 2014
}

\author{
For a complete list of Discussion Papers published by the SFB 649, \\ please visit http://sfb649.wiwi.hu-berlin.de.
}

019 "Unemployment benefits extensions at the zero lower bound on nominal interest rate" by Julien Albertini and Arthur Poirier, February 2014.

020 "Modelling spatio-temporal variability of temperature" by Xiaofeng Cao, Ostap Okhrin, Martin Odening and Matthias Ritter, February 2014.

021 "Do Maternal Health Problems Influence Child's Worrying Status? Evidence from British Cohort Study" by Xianhua Dai, Wolfgang Karl Härdle and Keming Yu, February 2014.

022 "Nonparametric Test for a Constant Beta over a Fixed Time Interval" by Markus Reiß, Viktor Todorov and George Tauchen, February 2014.

023 "Inflation Expectations Spillovers between the United States and Euro Area" by Aleksei Netšunajev and Lars Winkelmann, March 2014.

024 "Peer Effects and Students' Self-Control" by Berno Buechel, Lydia Mechtenberg and Julia Petersen, April 2014.

025 "Is there a demand for multi-year crop insurance?" by Maria Osipenko, Zhiwei Shen and Martin Odening, April 2014.

026 "Credit Risk Calibration based on CDS Spreads" by Shih-Kang Chao, Wolfgang Karl Härdle and Hien Pham-Thu, May 2014.

027 "Stale Forward Guidance" by Gunda-Alexandra Detmers and Dieter Nautz, May 2014.

028 "Confidence Corridors for Multivariate Generalized Quantile Regression" by Shih-Kang Chao, Katharina Proksch, Holger Dette and Wolfgang Härdle, May 2014.

029 "Information Risk, Market Stress and Institutional Herding in Financial Markets: New Evidence Through the Lens of a Simulated Model" by Christopher Boortz, Stephanie Kremer, Simon Jurkatis and Dieter Nautz, May 2014.

030 "Forecasting Generalized Quantiles of Electricity Demand: A Functional Data Approach" by Brenda López Cabrera and Franziska Schulz, May 2014.

031 "Structural Vector Autoregressions with Smooth Transition in Variances The Interaction Between U.S. Monetary Policy and the Stock Market" by Helmut Lütkepohl and Aleksei Netsunajev, June 2014.

032 "TEDAS - Tail Event Driven ASset Allocation" by Wolfgang Karl Härdle, Sergey Nasekin, David Lee Kuo Chuen and Phoon Kok Fai, June 2014.

033 "Discount Factor Shocks and Labor Market Dynamics" by Julien Albertini and Arthur Poirier, June 2014.

034 "Risky Linear Approximations" by Alexander Meyer-Gohde, July 2014

035 "Adaptive Order Flow Forecasting with Multiplicative Error Models" by Wolfgang Karl Härdle, Andrija Mihoci and Christopher Hian-Ann Ting, July 2014

036 "Portfolio Decisions and Brain Reactions via the CEAD method" by Piotr Majer, Peter N.C. Mohr, Hauke R. Heekeren and Wolfgang K. Härdle, July 2014

037 "Common price and volatility jumps in noisy high-frequency data" by Markus Bibinger and Lars Winkelmann, July 2014

038 "Spatial Wage Inequality and Technological Change" by Charlotte Senftleben-König and Hanna Wielandt, August 2014

039 "The integration of credit default swap markets in the pre and postsubprime crisis in common stochastic trends" by Cathy Yi-Hsuan Chen, Wolfgang Karl Härdle, Hien Pham-Thu, August 2014

\section{SFB 649, Spandauer Straße 1, D-10178 Berlin http://sfb649.wiwi.hu-berlin.de}

This research was supported by the Deutsche Forschungsgemeinschaft through the SFB 649 "Economic Risk".

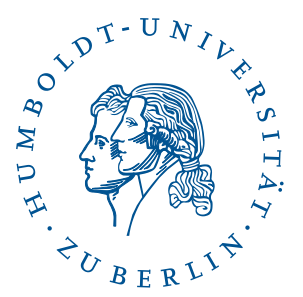




\section{SFB 649 Discussion Paper Series 2014}

For a complete list of Discussion Papers published by the SFB 649, please visit http://sfb649.wiwi.hu-berlin.de.

040 "Localising Forward Intensities for Multiperiod Corporate Default" by Dedy Dwi Prastyo and Wolfgang Karl Härdle, August 2014.

041 "Certification and Market Transparency" by Konrad Stahl and Roland Strausz, September 2014.

042 "Beyond dimension two: A test for higher-order tail risk" by Carsten Bormann, Melanie Schienle and Julia Schaumburg, September 2014.

043 "Semiparametric Estimation with Generated Covariates" by Enno Mammen, Christoph Rothe and Melanie Schienle, September 2014.

044 "On the Timing of Climate Agreements" by Robert C. Schmid and Roland Strausz, September 2014.

045 "Optimal Sales Contracts with Withdrawal Rights" by Daniel Krähmer and Roland Strausz, September 2014.

046 "Ex post information rents in sequential screening" by Daniel Krähmer and Roland Strausz, September 2014. 